\title{
TOPSIS Method Based on Complex Spherical Fuzzy Sets with Bonferroni Mean Operators
}

\author{
Zeeshan Ali ${ }^{1}$, Tahir Mahmood ${ }^{1}$ (D) and Miin-Shen Yang ${ }^{2, *}$ \\ 1 Department of Mathematics and Statistics, International Islamic University, Islamabad 44000, Pakistan; \\ zeeshan.phdma102@iiu.edu.pk (Z.A.); tahirbakhat@iiu.edu.pk (T.M.) \\ 2 Department of Applied Mathematics, Chung Yuan Christian University, Chung-Li 32023, Taiwan \\ * Correspondence: msyang@math.cycu.edu.tw
}

Received: 14 September 2020; Accepted: 5 October 2020; Published: 10 October 2020

\begin{abstract}
The theory of complex spherical fuzzy sets (CSFSs) is a mixture of two theories, i.e., complex fuzzy sets (CFSs) and spherical fuzzy sets (SFSs), to cope with uncertain and unreliable information in realistic decision-making situations. CSFSs contain three grades in the form of polar coordinates, e.g., truth, abstinence, and falsity, belonging to a unit disc in a complex plane, with a condition that the sum of squares of the real part of the truth, abstinence, and falsity grades is not exceeded by a unit interval. In this paper, we first consider some properties and their operational laws of CSFSs. Additionally, based on CSFSs, the complex spherical fuzzy Bonferroni mean (CSFBM) and complex spherical fuzzy weighted Bonferroni mean (CSFWBM) operators are proposed. The special cases of the proposed operators are also discussed. A multi-attribute decision making (MADM) problem was chosen to be resolved based on the proposed CSFBM and CSFWBM operators. We then propose the Technique for Order Preference by Similarity to an Ideal Solution (TOPSIS) method based on CSFSs (CSFS-TOPSIS). An application example is given to delineate the proposed methods and a close examination is undertaken. The advantages and comparative analysis of the proposed approaches are also presented.
\end{abstract}

Keywords: fuzzy sets; complex spherical fuzzy sets; Bonferroni mean operators; TOPSIS method

\section{Introduction}

Multi-attribute decision making (MADM) issues are inescapable in the field of decision making. In numerous functional applications, MADM plays a significant role in the procedure of decision making. Many existing strategies tell us that the best way to pick the most appropriate elective depends on the decision makers' (DMs) assessment data. Due to the progressively intricate outer decision-making condition and the abstract vulnerability of DMs themselves, it is hard for DMs to clarify their genuine inclination data plainly. In this manner, Zadeh [1] characterized the idea of fuzzy sets (FSs) to clarify the imprecision and the doubt occurring during the assessment procedure. Until now, FSs have been examined and applied to different fields by a large number of scientists [2-4]. Later, numerous researchers have concentrated on the most proficient method to characterize the appraisal inclinations communicated by DMs more extensively and precisely. Numerous categories of FSs have been proposed to adjust to different application conditions, for example, intuitionistic FSs (IFSs) explored by Atanassov [5] contain supporting and non-supporting grades with a rule that the sum of both cannot be exceeded from a unit interval.

However, the condition of an IFS for a decision maker is somehow too restrictive for choosing the sum of supporting and non-supporting grades that is not exceeded from a unit interval. To resolve such issues, the theory of Pythagorean fuzzy sets (PFSs) was explored by Yager [6], with a condition that the sum of the squares of both cannot be exceeded from a unit interval. IFSs and PFSs have had 
various applications [7-12]. Later, the theory of picture fuzzy sets (PiFSs) was presented by Cuong and Kreinovich [13]. PiFSs are composed of the grades of truth, abstinence, and falsity with a condition that the sum of all grades cannot be exceeded from a unit interval. Similarly, the condition of PiFSs for a decision maker is also too restrictive for choosing the sum of truth, abstinence, and falsity grades that is not exceeded from a unit interval. Thus, the theory of spherical fuzzy set (SFS), proposed by Mahmood et al. [14], with a condition that the sum of squares of all grades cannot be exceeded from a unit interval, was used to resolve these issues. By extending squares with q-powers, T-spherical fuzzy sets (TSFSs) were established by Ullah et al. [15], in which the sum of q-powers of positive, abstinence, and negative grades belong to [0,1] with various applications in different fields [16-20].

From the above studies of decision maker processes, we can conclude that their introduction is constrained and can deal with only with vulnerability in information, yet at the same time neglects to manage changes at a given period of time. Be that as it may, data obtained, such as from a clinical examination, or a database for biometric and facial acknowledgment, consistently changes simultaneously with time. Along these lines, to manage such sorts of issue, the scope of a supporting grade is arrived at from a genuine subset to the unit plate of the mind boggling plane and thus Ramot et al. [21] established the complex FS (CFS) which has had many applications [22-24]. Additionally, the theory of complex IFSs (CIFSs) was presented by Alkouri and Salleh [25] to provide a wide range of options to a decision maker for taking a decision. CIFSs compose the supporting grade and the non-supporting grade in the form of a complex number belonging to a unit disc in a complex plane. The limitations of CIFSs is that the sum of the real part (and the imaginary part) of both grades cannot be exceeded from a unit interval. However, a decision maker may give the grades of both real and imaginary parts whose sum is exceeded from a unit interval. The theory of complex PFSs (CPFSs), with a condition in which the sum of squares of the grades of both real and imaginary parts cannot be exceeded from a unit interval, was proposed by Ullah et al. [26] for coping with this kind of issue. The theory of CIFS and CPFS have received lots of attention with applications in different fields [27-30].

When a decision maker faces more types of answer, such as truly, abstinence, no, or refusal in the form of complex numbers, casting a ballot can be a genuine case in such a circumstance, as voters might be separated into four categories of individuals, i.e., vote in favor of, abstinence, vote against, or refusal of the democratic process, in the form of polar coordinates. For instance, with $\left(0.7 \mathrm{e}^{\mathrm{i} 2 \Pi 0.6}, 0.4 \mathrm{e}^{\mathrm{i} 2 \Pi 0.5}, 0.1 \mathrm{e}^{\mathrm{i} 2 \Pi 0.1}\right)$, the IFS, PFS, PFS, CIFS, or CPFS are not able to investigate, because the conditions of all these notions are limited. For coping with such issues, the theory of complex spherical fuzzy sets (CSFSs) is explored in this paper to examine proficiency and ability. Thus, we summarize the contributions of the paper as follows:

1. To investigate the novelty of CSFS and their fundamental laws.

2. To investigate the Bonferroni mean (BM) operators based on CSFS and discuss their special cases.

3. To examine the TOPSIS method based on CSFS and propose a novel CSFS-TOPSIS method.

4. To resolve the MADM issues based on the proposed aggregation operators.

5. To give an application example of the proposed methods with comparative analysis and demonstrate the usefulness and effectiveness of the proposed methods.

The remainder of the paper is organized as follows. In Section 2, we first review some basic definitions of CPFSs and BM operators, and then their score and accuracy function. We further consider their operational laws with some properties. In Section 3, based on CSFS and BM operators, the complex spherical fuzzy Bonferroni mean (CSFBM) and complex spherical fuzzy weighted Bonferroni mean (CSFWBM) operators are proposed. The special cases of the explored operators are also discussed to improve the novelty of the presented work. In Section 4, a MADM problem is chosen to be resolved based on the CSFBM operator and CSFWBM operator. Additionally, the TOPSIS method based on CSFS is also explored to construct a CSFS-TOPSIS method. An application example is given to demonstrate the effectiveness of the proposed methods with comparative analysis. Finally, we give conclusions in Section 5 . 


\section{Preliminary Definitions with Some Properties}

In this section, we review some existing notions, such as CPFSs, CSFSs and Bonferroni mean (BM) operators. Throughout the whole paper, we use $U_{\text {Univsersal }}$ to represent the universal set.

Definition 1 ([26]). A CPFS is defined as:

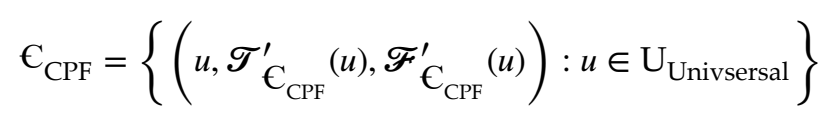

where $\boldsymbol{T}_{\epsilon_{C S F}}^{\prime}=\boldsymbol{T}_{\epsilon_{R P}} e^{i 2 \Pi \Omega_{S_{T}}}$ and $\mathcal{F}_{\epsilon_{C S F}}^{\prime}=\mathcal{F}_{\epsilon_{R P}} e^{i 2 \Pi \Omega_{\mathcal{F}_{C}}}$ expresses the grade of supporting and supporting against with the conditions: $0 \leq \boldsymbol{T}_{\epsilon_{R P}}{ }^{2}(u)+\mathcal{F}_{\epsilon_{R P}}{ }^{2}(u) \leq 1$ and $0 \leq \boldsymbol{\Omega}_{\mathcal{T}_{\epsilon_{I P}}}{ }^{2}(u)+$ $\boldsymbol{\Omega}_{\mathcal{F}_{C_{I P}}}{ }^{2}(u) \leq 1$. Further, the symbol $H_{C_{C S F}}(u)=\boldsymbol{\mu}_{C_{R P}} e^{i 2 \Pi \Omega_{\mu_{C_{I P}}}}=\left(1-\left(\boldsymbol{\mathcal { T }}_{\epsilon_{R P}}{ }^{2}(u)+\right.\right.$ $\left.\left.\mathcal{F}_{C_{R P}}{ }^{2}(u)\right)\right)^{\frac{1}{2}} e^{i 2 \Pi\left(1-\left(\Omega_{\mathcal{C}_{C_{I P}}}{ }^{2}(u)+\Omega_{\mathcal{F}_{C_{I P}}}{ }^{2}(u)\right)\right)^{\frac{1}{2}}}$ is called the hesitancy grade. In general, $\epsilon_{C P F}=\left(\mathcal{T}_{C_{C P F}}^{\prime}, \mathcal{F}_{C_{C P F}}^{\prime}\right)=$

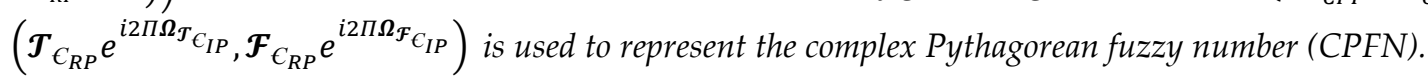

Definition 2 ([26]). For any CPFS $\epsilon_{C P F}$ with its CPFN $\epsilon_{C P F}=\left(\mathscr{T}^{\prime} \epsilon_{C P F}, \mathscr{F}^{\prime} \epsilon_{C P F}\right)=$ $\left(\mathscr{T}_{\mathrm{C}_{R P}} e^{i 2 \Pi \Omega \mathscr{T}} \mathrm{C}_{I P}, \mathscr{F} \mathrm{C}_{R P} e^{i 2 \Pi \Omega \mathscr{F}} \mathrm{C}_{I P}\right)$, the score function $S_{S F}$ and accuracy function $H_{A F}$ are defined as:

$$
\begin{aligned}
& S_{S F}\left(\epsilon_{C P F}\right)=\frac{1}{2}\left(\left(\mathcal{T}_{\epsilon_{R P}}^{2}-\mathcal{F}_{\epsilon_{R P}}^{2}\right)+\left(\boldsymbol{\Omega}_{\mathcal{T}_{I P}}^{2}-\boldsymbol{\Omega}_{\mathcal{F}_{C_{I P}}}^{2}\right)\right) \\
& H_{A F}\left(\epsilon_{C P F}\right)=\frac{1}{2}\left(\left(\boldsymbol{\mathcal { T }}_{\epsilon_{R P}}^{2}+\mathcal{F}_{\epsilon_{R P}}^{2}\right)+\left(\boldsymbol{\Omega}_{\mathcal{T}_{C_{I P}}}^{2}+\boldsymbol{\Omega}_{\mathcal{F}_{C_{I P}}}^{2}\right)\right)
\end{aligned}
$$

where $S_{S F}\left(\epsilon_{C P F}\right), H_{A F}\left(\epsilon_{C P F}\right) \in[-1,1]$.

Thus, the comparisons between two CPFNs $\epsilon_{C P F-1}$ and $\epsilon_{C P F-2}$ can be defined as:

1. If $S_{S F}\left(C_{C P F-1}\right)>S_{S F}\left(\epsilon_{C P F-2}\right)$, then $\mathrm{C}_{C P F-1}>\mathrm{C}_{C P F-2}$;

2. If $S_{S F}\left(C_{C P F-1}\right)=S_{S F}\left(C_{C P F-2}\right)$, then

1) If $H_{A F}\left(\epsilon_{C P F-1}\right)>H_{A F}\left(\epsilon_{C P F-2}\right)$, then $\epsilon_{C P F-1}>\epsilon_{C P F-2}$;

2) If $H_{A F}\left(\epsilon_{C P F-1}\right)=H_{A F}\left(\epsilon_{C P F-2}\right)$, then $\epsilon_{C P F-1}=\epsilon_{C P F-2}$.

Furthermore, we can give some important operators on $\mathrm{CPFNs}$, which are considered as follows. For any two CPFNs $\epsilon_{C P F-1}$ and $\epsilon_{C P F-2}$ with $s_{C S F}$, we have

1. $\epsilon_{C P F-1}{ }^{\mathrm{c}}=\left(\mathcal{F}_{\mathrm{C}_{R P-1}} \mathrm{e}^{\mathrm{i} 2 \Pi \boldsymbol{\Omega}_{\mathcal{F}_{I P-1}}, \boldsymbol{T}_{\mathrm{C}_{R P-1}}} \mathrm{e}^{\mathrm{i} 2 \pi \boldsymbol{\Omega}_{\mathcal{T}_{I P-1}}}\right)$;

2. $\epsilon_{C P F-1} \vee \epsilon_{C P F-2}=$

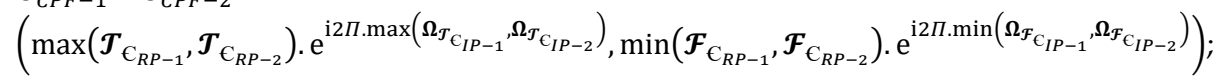

3. $\mathrm{\epsilon}_{C P F-1} \wedge \mathrm{\epsilon}_{C P F-2}=$

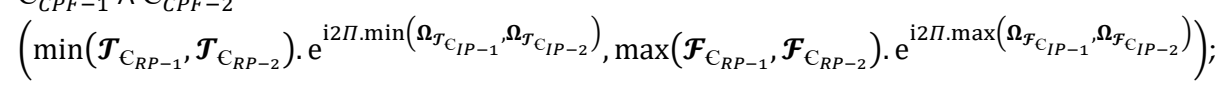




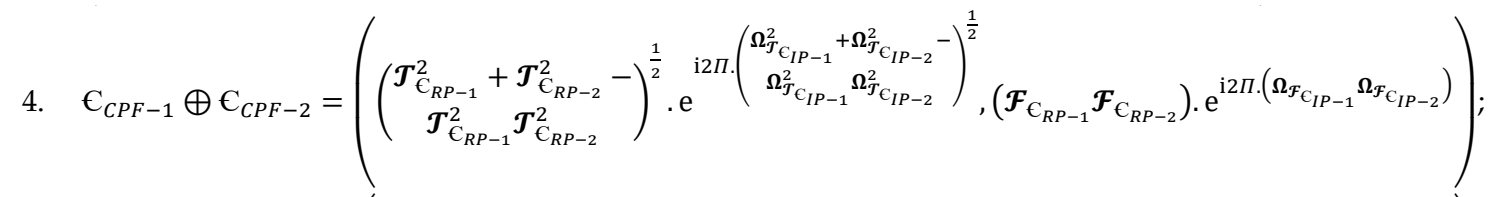

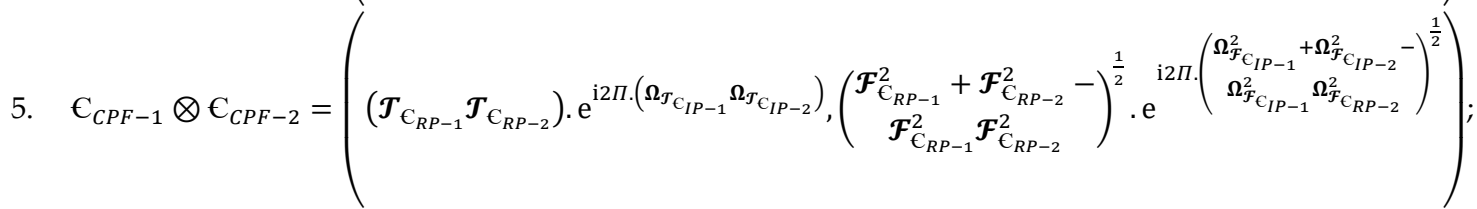

6. $\left.\left.\quad S_{P F} \mathrm{C}_{C P F-1}=\left(\left(1-\left(1-\boldsymbol{T}_{\mathrm{C}_{R P-1}}^{2}\right)^{s_{C S F}}\right)^{\frac{1}{2}} \mathrm{e}^{\mathrm{i} 2 \Pi \cdot\left(1-\left(1-\Omega_{\mathcal{T}_{C I P-1}}^{2}\right.\right.}\right)^{s_{C S F}}\right)^{\frac{1}{2}}, \mathcal{F}_{\mathrm{C}_{R P-1}}^{s_{C S F}} \mathrm{e}^{\mathrm{i} 2 \Pi \Omega_{\mathcal{F}_{I P-1}}^{s_{C S F}}}\right)$

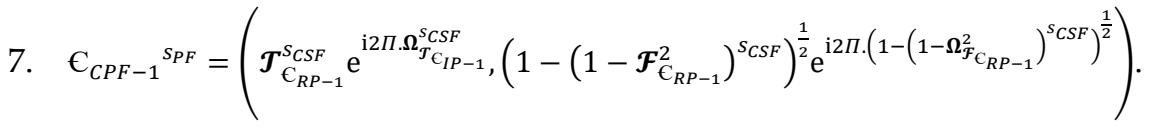

Definition 3 ([31]). For any collection $\left\{V_{j}, j=1,2,3, \ldots, m\right\}$ of values with $0 \leq V_{j} \leq 1$, and $0 \leq p, q$, the $B M$ operator is defined as:

$$
B M^{p, q}\left(V_{1}, V_{2}, \ldots, V_{m}\right)=\left(\frac{1}{m(m-1)} \sum_{\substack{j, k=1 \\ j \neq k}}^{m} V_{j}^{p} V_{k}^{q}\right)^{\frac{1}{p+q}}
$$

We next give the definition of CSFSs and their operational laws. Ali et al. [32] recently proposed complex T-spherical fuzzy sets (CTSFSs). In fact, a CSFS is a special case of a CTSFS with $q=2$. The definition of a CSFS is given as follows.

Definition 4 ([32]). A CSFS is defined as:

$$
\epsilon_{C S F}=\left\{\left(u, \boldsymbol{T}_{\epsilon_{C S F}}^{\prime}(u), \boldsymbol{\theta}_{\epsilon_{C S F}}^{\prime}(u), \mathcal{F}_{\epsilon_{C S F}}^{\prime}(u)\right): u \in U_{\text {Universal }}\right\}
$$

where $\boldsymbol{T}_{C_{C S F}}^{\prime}=\boldsymbol{T}_{C_{R P}} e^{i 2 \Pi \boldsymbol{\Omega}_{C_{I P}}}, \boldsymbol{\theta}_{C_{C S F}}^{\prime}=\boldsymbol{\theta}_{C_{R P}} e^{i 2 \Pi \boldsymbol{\Omega}_{\epsilon_{I P}}}$ and $\mathcal{F}_{C_{C S F}}^{\prime}=\mathcal{F}_{C_{R P}} e^{i 2 \Pi \boldsymbol{\Omega}_{\mathcal{F}_{I P}}}$ expresses the grade of supporting, abstinence, and supporting against with the conditions: $0 \leq \boldsymbol{\mathcal { T }}_{\epsilon_{R P}}{ }^{2}(u)+\boldsymbol{\theta}_{\epsilon_{R P}}^{2}(u)+$ $\mathcal{F}_{\epsilon_{R P}}{ }^{2}(u) \leq 1$ and $0 \leq \boldsymbol{\Omega}_{\mathcal{T}_{C_{I P}}}{ }^{2}(u)+\boldsymbol{\Omega}_{\boldsymbol{\theta}_{C_{I P}}}{ }^{2}(u)+\boldsymbol{\Omega}_{\mathcal{F}_{I P}}{ }^{2}(u) \leq 1$. Further, the symbol $H_{C_{C S F}}(u)=$ $\boldsymbol{\mu}_{C_{R P}} e^{i 2 \Pi \Omega_{\mu_{I P}}}=\left(1-\left(\boldsymbol{\mathcal { T }}_{C_{R P}}{ }^{2}(u)+\boldsymbol{\theta}_{C_{R P}}^{2}(u)+\boldsymbol{\mathcal { F }}_{C_{R P}}{ }^{2}(u)\right)\right)^{\frac{1}{2}} e^{i 2 \Pi\left(1-\left(\Omega_{\mathcal{T}_{C_{I P}}}{ }^{2}(u)+\boldsymbol{\Omega}_{\theta_{C_{I P}}}{ }^{2}(u)+\Omega_{\mathcal{F}_{I P}}{ }^{2}(u)\right)\right)^{\frac{1}{2}}}$ is called the hesitancy grade. Thus, $\epsilon_{C S F}=\left(\mathcal{T}_{\epsilon_{C S F}}^{\prime}, \boldsymbol{\theta}_{\epsilon_{C S F}}^{\prime}, \mathcal{F}_{\epsilon_{C S F}}^{\prime}\right)=$ $\left(\boldsymbol{\mathcal { T }}_{\epsilon_{R P}} e^{i 2 \Pi \Omega_{\mathcal{T}}} \epsilon_{I P}, \boldsymbol{\theta}_{\epsilon_{R P}} e^{i 2 \Pi \Omega_{\epsilon_{I P}}, \mathcal{F}_{C_{R P}}} e^{i 2 \Pi \Omega_{\mathcal{F}}}{ }_{C_{I P}}\right)$ represents the complex spherical fuzzy number (CSFN).

Definition 5. For any CSFS $\epsilon_{C S F}$ with its CSFN $\epsilon_{C S F}=\left(\mathscr{T}_{\mathcal{C}_{C S F}^{\prime}}^{\prime}, \theta_{\mathcal{C}_{C S F}}^{\prime}, \mathscr{F}^{\prime} \in_{C S F}\right)=$ $\left(\mathscr{T}_{\mathrm{C}_{R P}} e^{i 2 \Pi \Omega_{\mathscr{T}}} \mathrm{C}_{I P}, \theta \mathrm{C}_{R P} e^{i 2 \Pi \Omega_{\theta}} \mathrm{C}_{I P}, \mathscr{F} \mathrm{C}_{R P} e^{i 2 \Pi \Omega_{\mathscr{F}}} \mathrm{C}_{I P}\right)$, the score function $S_{S F}$ and accuracy function $H_{A F}$ is defined as:

$$
S_{S F}\left(\epsilon_{C S F}\right)=\frac{1}{3}\left(\left(\mathcal{T}_{\epsilon_{R P}}^{2}-\boldsymbol{\theta}_{\epsilon_{R P}}^{2}-\mathcal{F}_{\epsilon_{R P}}^{2}\right)+\left(\boldsymbol{\Omega}_{\mathcal{T}_{C_{I P}}}^{2}-\boldsymbol{\Omega}_{\boldsymbol{\theta}_{C_{I P}}}^{2}-\boldsymbol{\Omega}_{\mathcal{F}_{C_{I P}}}^{2}\right)\right)
$$




$$
H_{A F}\left(\epsilon_{C S F}\right)=\frac{1}{3}\left(\left(\boldsymbol{\mathcal { T }}_{\epsilon_{R P}}^{2}+\boldsymbol{\theta}_{\epsilon_{R P}}^{2}+\mathcal{F}_{\epsilon_{R P}}^{2}\right)+\left(\boldsymbol{\Omega}_{\mathcal{T}_{C_{I P}}}^{2}+\boldsymbol{\Omega}_{\boldsymbol{\theta}_{C_{I P}}}^{2}+\boldsymbol{\Omega}_{\mathcal{F}_{C_{I P}}}^{2}\right)\right)
$$

where $S_{S F}\left(\mathrm{C}_{C S F}\right), H_{A F}\left(\mathrm{C}_{C S F}\right) \in[-1,1]$.

Thus, the comparisons between two CSFNs $\epsilon_{C S F-1}$ and $\epsilon_{C S F-2}$ can be stated as:

1. If $S_{S F}\left(\epsilon_{C S F-1}\right)>S_{S F}\left(\epsilon_{C S F-2}\right)$, then $\mathrm{C}_{C S F-1}>\epsilon_{C S F-2}$

2. If $S_{S F}\left(\epsilon_{C S F-1}\right)=S_{S F}\left(\epsilon_{C S F-2}\right)$, then

1) If $H_{A F}\left(\epsilon_{C S F-1}\right)>H_{A F}\left(\epsilon_{C S F-2}\right)$, then $\epsilon_{C S F-1}>\epsilon_{C S F-2}$

2) If $H_{A F}\left(\epsilon_{C S F-1}\right)=H_{A F}\left(\epsilon_{C S F-2}\right)$, then $\epsilon_{C S F-1}=\epsilon_{C S F-2}$.

We next give some important operators of CSFNs. For any two CSFNs $\epsilon_{C S F-1}$ and $\epsilon_{C S F-2}$ with $s_{C S F}$, we give the following operators of CSFNs:

1. $\epsilon_{C S F-1}{ }^{c}=\left(\mathcal{F}_{C_{R P-1}} e^{i 2 \Pi \Omega_{\mathcal{F}}}{ }_{I P-1}, \boldsymbol{\theta}_{C_{R P-1}} e^{i 2 \Pi \Omega_{C_{I P-1}}, \boldsymbol{T}_{\epsilon_{R P-1}}} e^{i 2 \Pi \Omega_{\mathcal{T}_{I P-1}}}\right)$;

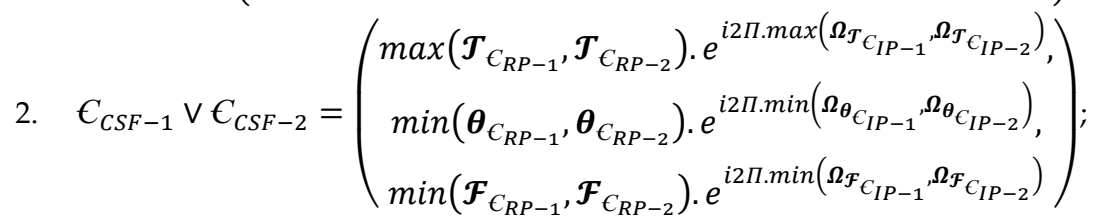

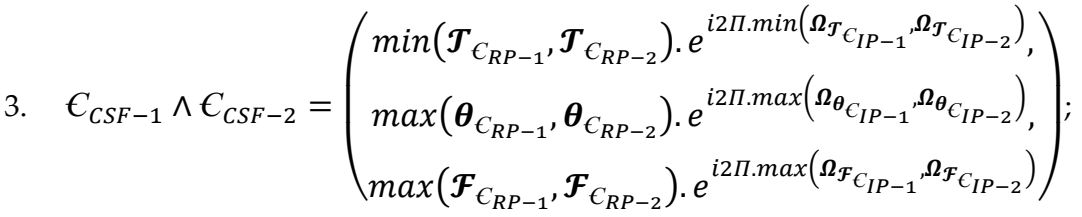

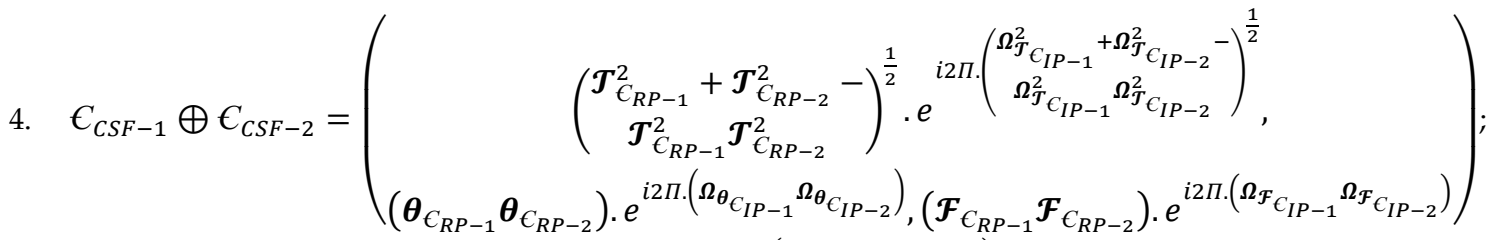

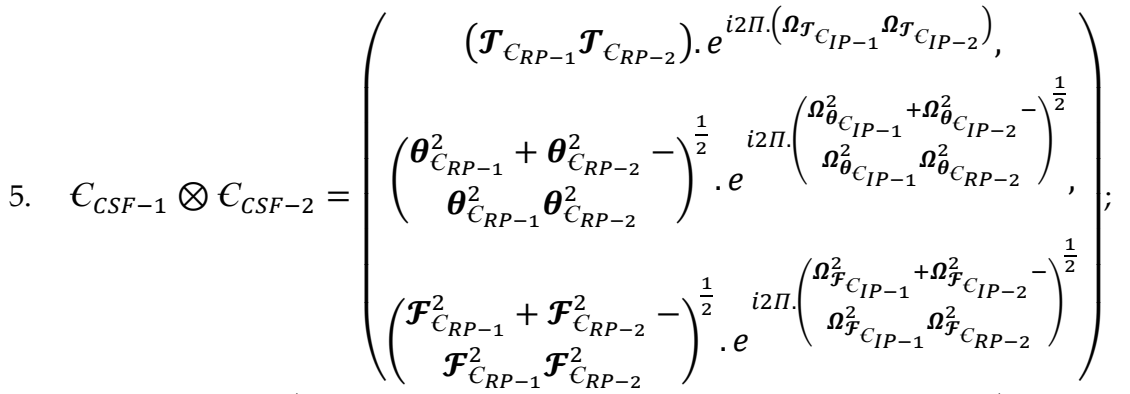

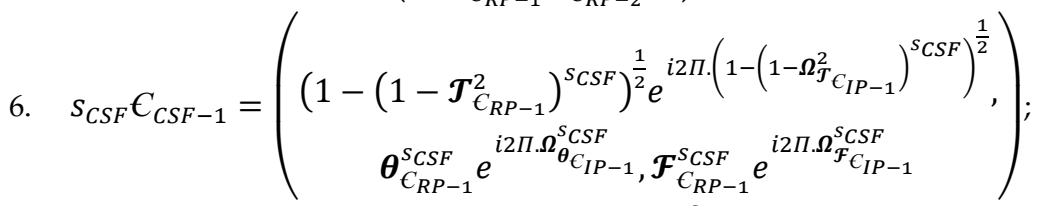

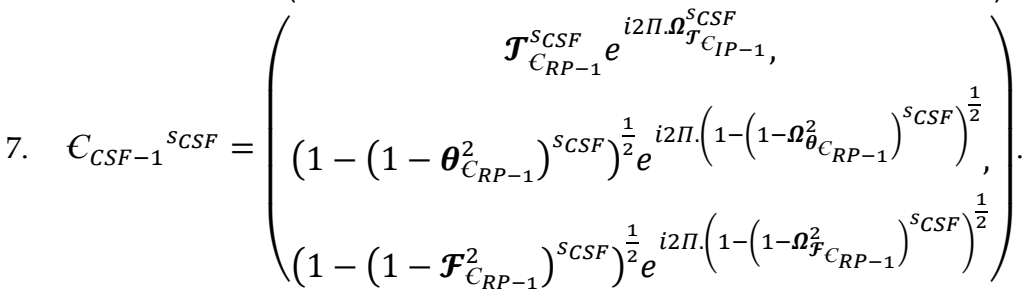




\section{The Bonferroni Mean Operators Based on CSFSs}

In this section, we give two important Bonferroni mean (BM) operators based on CSFSs, called CSFBM and CSFWBM. Further, the specific cases of the CSFBM operator are also justified with some remarks.

Definition 6. For any CSFSS $\mathscr{C}_{C S F-j}, \mathbf{j = 1} \mathbf{1}, \mathbf{3}, . ., m$, the CSFBM operator is defined as:

$$
\begin{aligned}
\operatorname{CSFBM}^{s_{C S F}, t_{C S F}}( & \left.\mathcal{C}_{C S F-1}, \mathcal{C}_{C S F-2}, \ldots, \mathcal{C}_{C S F-m}\right) \\
& =\left(\frac{1}{m(m-1)} \bigoplus_{\substack{j, k=1 \\
j \neq k}}^{m}\left(\mathcal{C}_{C S F-j}^{s_{C S F}} \otimes \mathcal{C}_{C S F-k}^{t_{C S F}}\right)\right)^{\frac{1}{s_{C S F}+t_{C S F}}}
\end{aligned}
$$

Theorem 1. The CSFBM operation result is still a CSFS, such that it has the following equation:

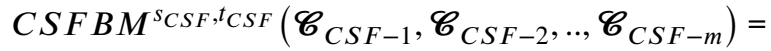

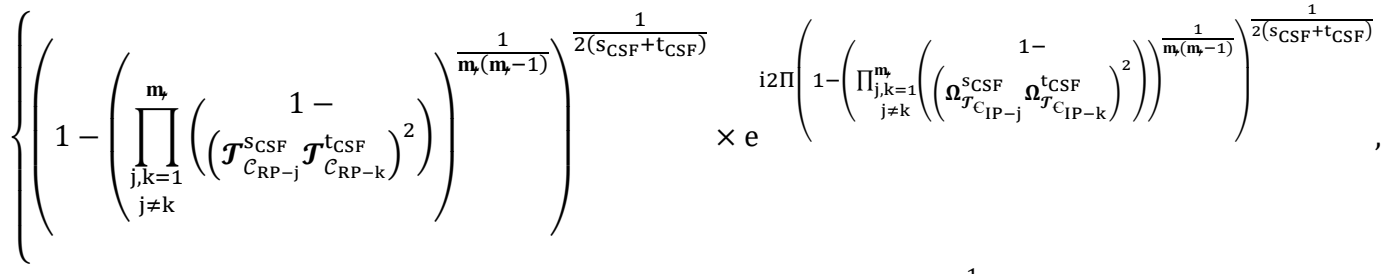

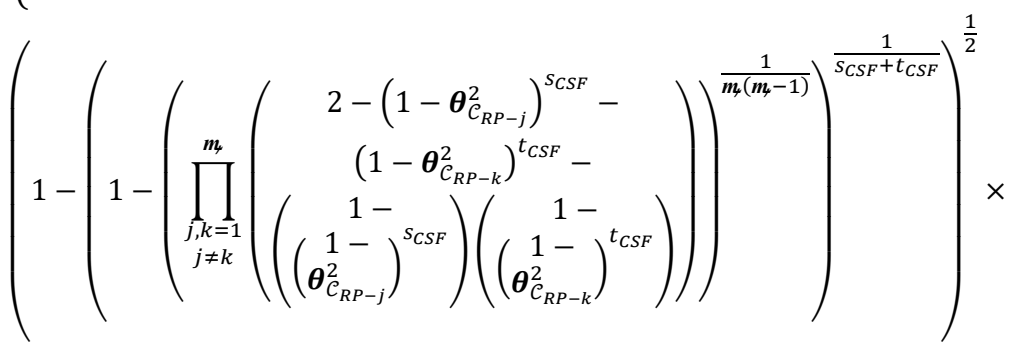

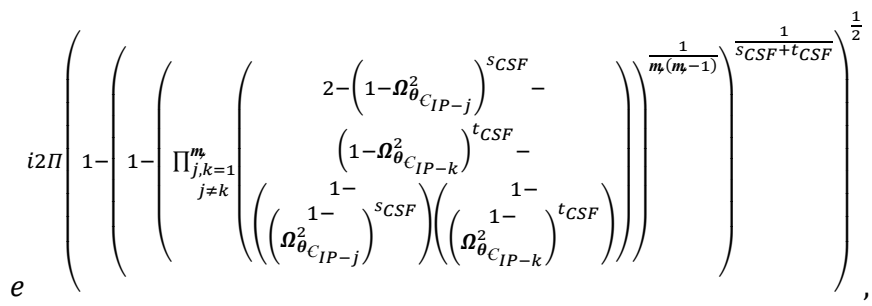

$$
\begin{aligned}
& \left.\left.1-\left(1-\left(\begin{array}{c}
\left.\prod_{\substack{j, k=1 \\
j \neq k}}^{m}\left(\begin{array}{c}
2-\left(1-\mathcal{F}_{\mathcal{C}_{R P-j}}^{2}\right)^{s_{C S F}}- \\
\left(1-\mathcal{F}_{\mathcal{C}_{R P-k}}^{2}\right)^{t_{C S F}-} \\
1-{ }^{1-} s_{C S F} \\
\left(\mathcal{F}_{\mathcal{C}_{R P-j}}^{2}\right.
\end{array}\right)\left(\begin{array}{c}
1- \\
\left(\begin{array}{c}
1- \\
\mathcal{F}_{\mathcal{C}_{R P-k}}^{2}
\end{array}\right)^{t_{C S F}}
\end{array}\right)\right)
\end{array}\right)\right)^{\frac{1}{m_{(m-1)}}}\right)^{\frac{1}{s_{C S F+}+t_{C F}}}\right)^{\frac{1}{2}} \times
\end{aligned}
$$

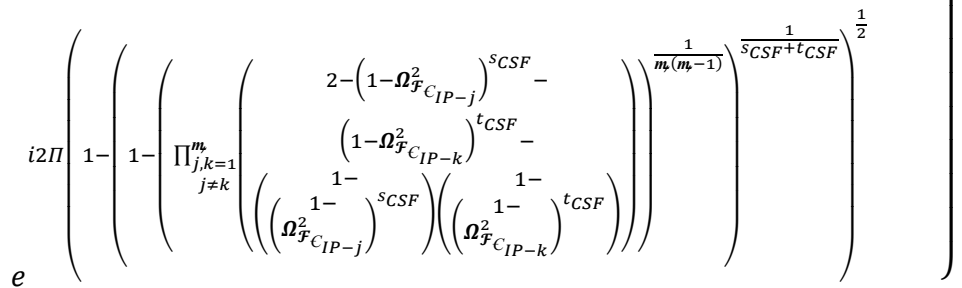




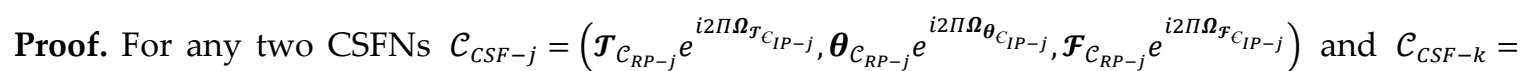
$\left(\boldsymbol{T}_{\mathcal{C}_{R P-k}} e^{i 2 \pi \Omega_{\mathcal{C}_{I P-k}}}, \boldsymbol{\theta}_{\mathcal{C}_{R P-k}} e^{i 2 \Pi \boldsymbol{\Omega}_{\boldsymbol{\theta}_{I P-k}}, \mathcal{F}_{C_{R P-k}}} e^{i 2 \Pi \boldsymbol{\Omega}_{\mathcal{F}_{I P-k}}}\right)$, based on the definition of CSFBM operator, we can get

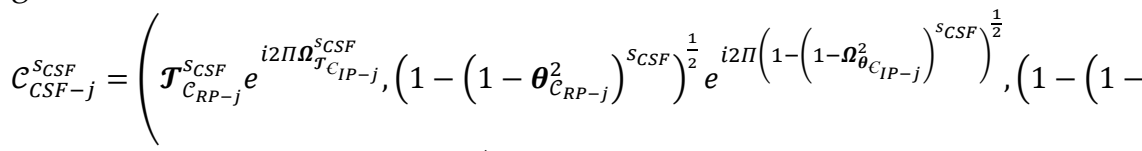
$\left.\left.\left.\left.\left.\mathcal{F}_{\mathcal{C}_{R P-j}}^{2}\right)^{s_{C S F}}\right)^{\frac{1}{2}} e^{i 2 \Pi\left(1-\left(1-\Omega_{\mathcal{F}_{C_{I P-j}}}^{2}\right.\right.}\right)^{s_{C S F}}\right)^{\frac{1}{2}}\right) \quad, \quad$ and $\quad \mathcal{C}_{C S F-k}^{t_{C S F}}=\left(\boldsymbol{T}_{\mathcal{C}_{R P-k}}^{t_{C S F}} e^{i 2 \pi \Omega_{T_{I P-k}}^{t_{C S F}},(1-(1-}\right.$ $\left.\left.\left.\left.\left.\boldsymbol{\theta}_{C_{R P-k}}^{2}\right)^{t_{C S F}}\right)^{\frac{1}{2}} e^{i 2 \Pi\left(1-\left(1-\Omega_{\theta_{I P-k}}^{2}\right.\right.}\right)^{t_{C S F}}\right)^{\frac{1}{2}},\left(1-\left(1-\mathcal{F}_{\mathcal{C}_{R P-k}}^{2}\right)^{t_{C S F}}\right)^{\frac{1}{2}} e^{i 2 \Pi\left(1-\left(1-\Omega_{C_{I P-k}}^{2}\right)^{t_{C S F}}\right)^{\frac{1}{2}}}\right)$. Then, we have

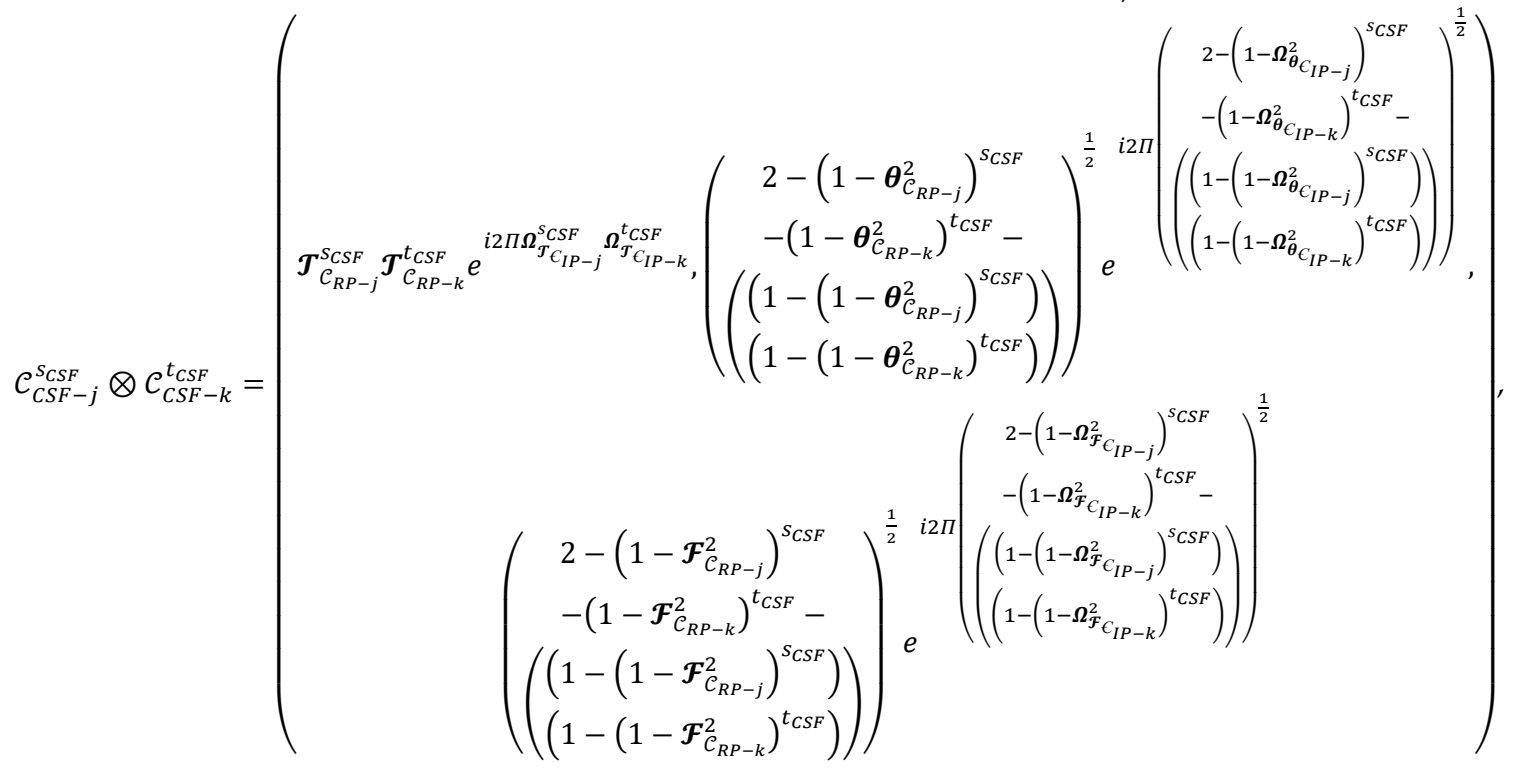

And

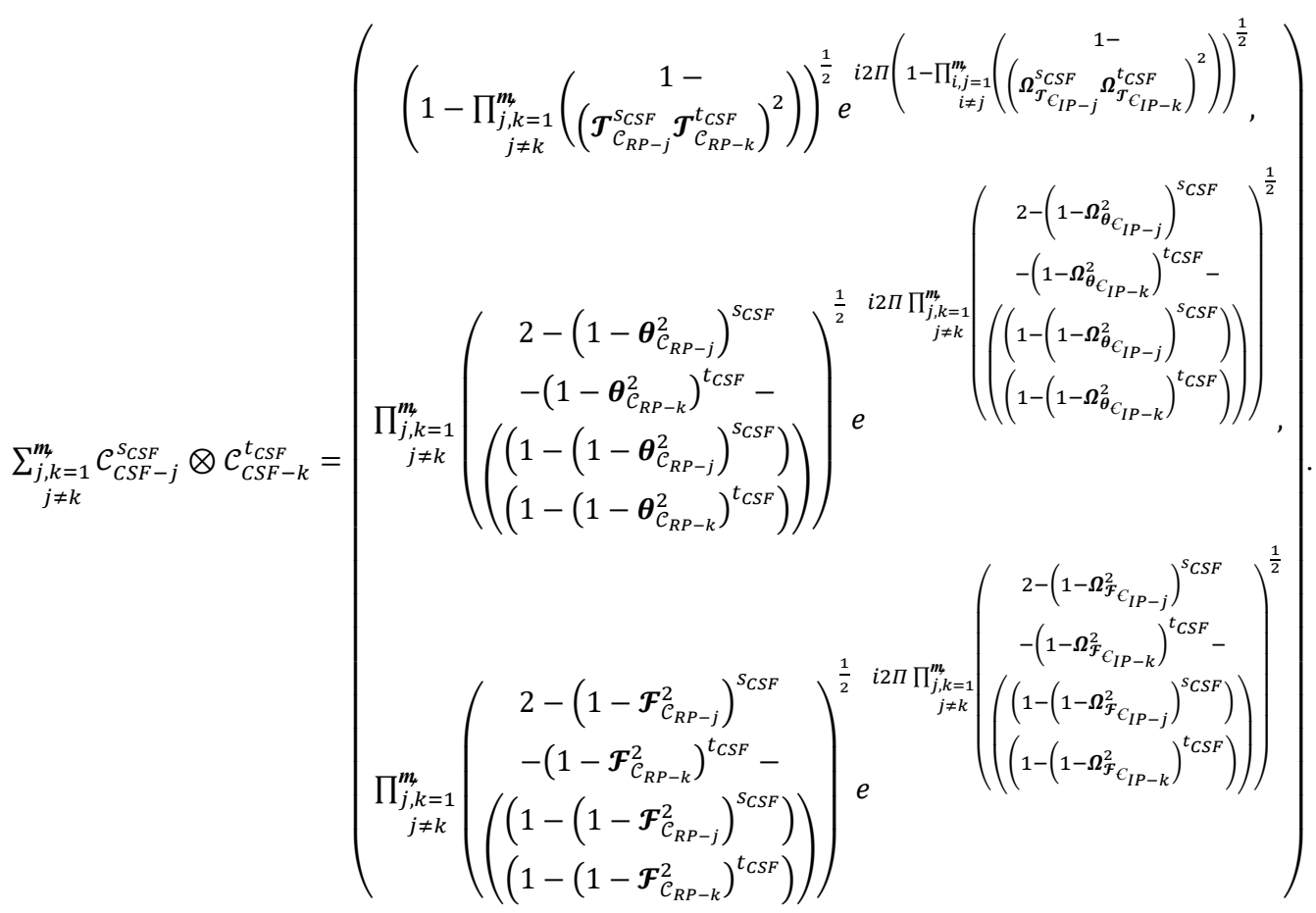


Thus, we have

$\frac{1}{\boldsymbol{m}_{\boldsymbol{H}}\left(\boldsymbol{m}_{\boldsymbol{H}}-1\right)} \sum_{\substack{j, k=1 \\ j \neq k}}^{\boldsymbol{m}_{\boldsymbol{F}}} \mathcal{C}_{C S F-j}^{S_{C S F}} \otimes \mathcal{C}_{C S F-k}^{t_{C S F}}$

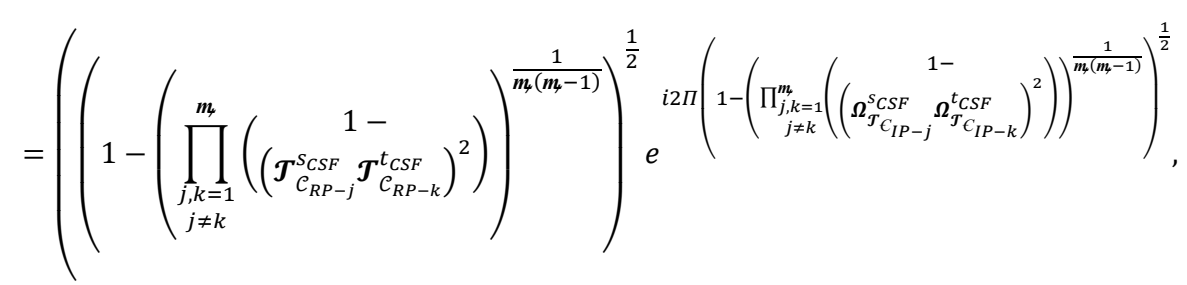

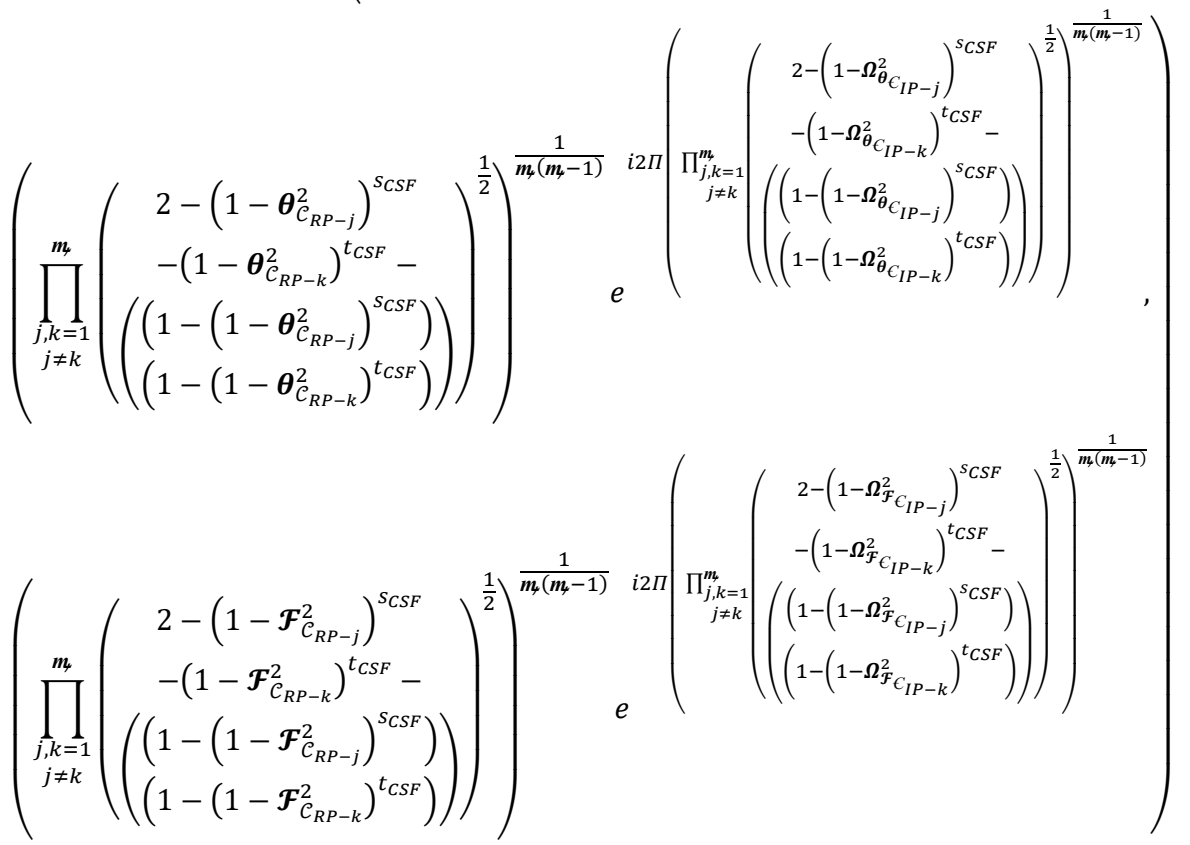

And

$$
\left(\frac{1}{\boldsymbol{m}_{\boldsymbol{y}}\left(\boldsymbol{m}_{\boldsymbol{q}}-1\right)} \bigoplus_{\substack{j, k=1 \\ j \neq k}}^{\boldsymbol{m}_{\boldsymbol{k}}}\left(\mathcal{C}_{C S F-j}^{S_{C S F}} \otimes \mathcal{C}_{C S F-k}^{t_{C S F}}\right)\right)^{\frac{1}{s_{C S F}+t_{C S F}}}=
$$

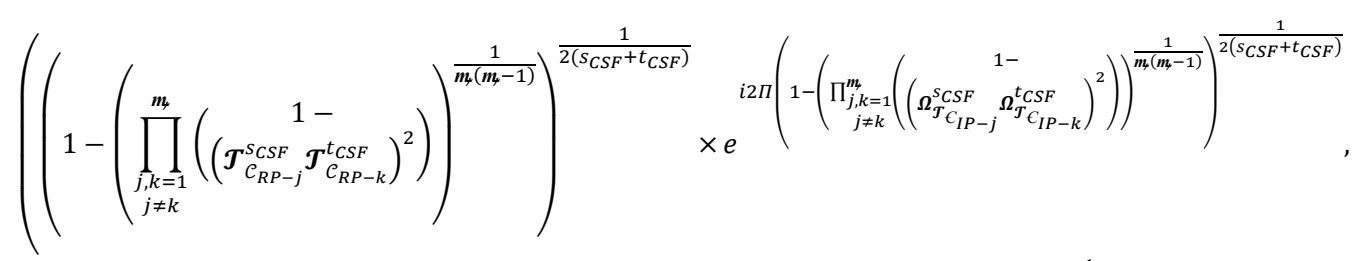

$$
\begin{aligned}
& \left.\left.1-\left(1-\left(\prod_{\substack{j, k=1 \\
j \neq k}}^{m_{*}}\left(\begin{array}{c}
2-\left(1-\boldsymbol{\theta}_{\mathcal{C}_{R P-j}}^{2}\right)^{s_{C S F}} \\
-\left(1-\boldsymbol{\theta}_{\mathcal{C}_{R P-k}}^{2}\right)^{t_{C S F}}- \\
\left(1-\left(1-\boldsymbol{\theta}_{\mathcal{C}_{R P-j}}^{2}\right)^{s_{C S F}}\right)
\end{array}\right)\right)\right)^{\frac{1}{\boldsymbol{m}_{(}\left(m_{-}-1\right)}}\right)^{\frac{1}{s_{C S F}+t_{C S F}}}\right)^{\frac{1}{2}}
\end{aligned}
$$




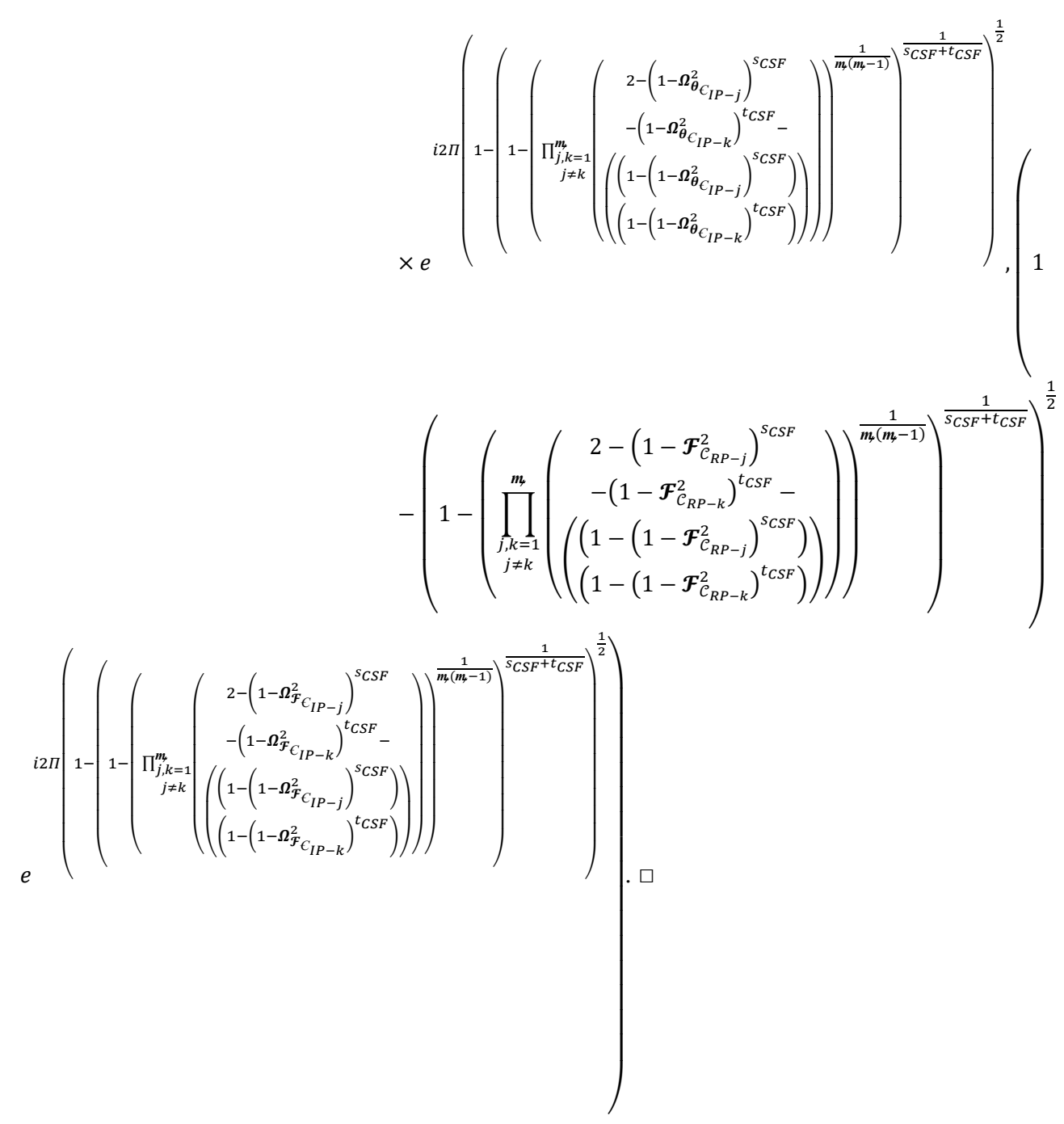

We next investigate the properties of idempotency, monotonicity, and boundedness for the CSFBM operator.

Theorem 2 (Idempotency). For any CSFSs $\mathscr{C}_{C S F-j}, j=1,2,3, . ., m$, we have

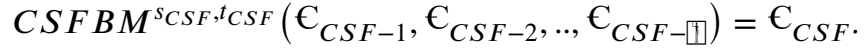

Proof. Suppose $C S F B M^{s_{C S F}, t_{C S F}}\left(\epsilon_{C S F-1}, \epsilon_{C S F-2}, \ldots, \epsilon_{C S F-m_{*}}\right)=(u, w, v)$. First, we choose the supporting grade with $\Phi_{\epsilon_{C S F}}^{\prime}=\Phi_{\epsilon_{R P}} e^{i 2 \Pi \Psi_{\Phi_{I P}}}$. Let $\Phi_{\epsilon_{R P}}=\Phi_{\epsilon_{R P-j}} \psi_{\Phi_{C_{I P}}}=\Psi_{\Phi_{\epsilon_{I P-j}}}$ and $\Phi_{\epsilon_{R P}}=$ $\Phi_{C_{R P-k}}, \Psi_{\Phi_{C_{I P}}}=\Psi_{\Phi_{C_{I P-k}}}$. Then, $\Phi_{C_{R P}} e^{i 2 \Pi \Psi_{\Phi_{I P}}}=\Phi_{C_{R P-j}} e^{i 2 \Pi \Psi_{C_{I P-j}}}$ and $\Phi_{C_{R P}} e^{i 2 \Pi \Psi_{\Phi_{I P}}}=\Phi_{C_{R P-k}} e^{i 2 \Pi \Psi_{\Phi_{I P-k}}}$, and 


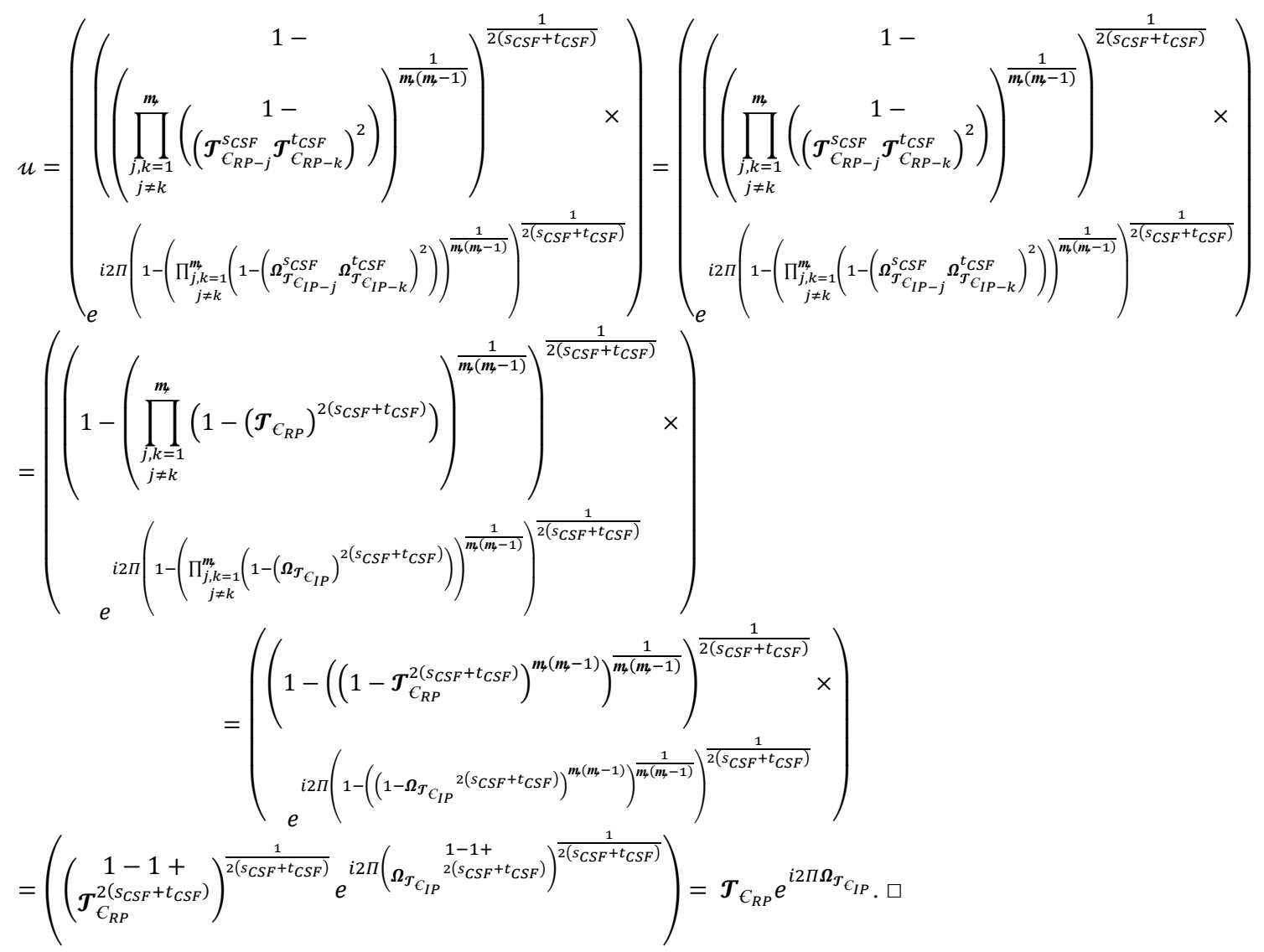

Similarly, we can examine for abstinence and non-supporting grades, such that $\boldsymbol{\theta}_{\epsilon_{C S F}}^{\prime}=$ $\boldsymbol{\theta}_{C_{R P}} e^{i 2 \Pi \Omega_{\boldsymbol{\theta}}} \epsilon_{I P}$ and $\mathcal{F}_{C_{C S F}}^{\prime}=\mathcal{F}_{C_{R P}} e^{i 2 \Pi \Omega_{\mathcal{F}}} \epsilon_{I P}$. Hence, $C S F B M^{S_{C S F}, t_{C S F}}\left(\epsilon_{C S F-1}, \epsilon_{C S F-2}, \ldots, \epsilon_{C S F-m_{4}}\right)=\epsilon_{C S F}$.

Theorem 3 (Monotonicity). For any two CSFSs

$\epsilon_{C S F-j}=\left(\boldsymbol{T}_{C_{R P-j}} e^{i 2 \Pi \Omega_{T_{I P-j}}}, \boldsymbol{\theta}_{C_{R P-j}} e^{i 2 \Pi \boldsymbol{\Omega}_{\epsilon_{I P-j}}, \mathcal{F}_{C_{R P-j}}} e^{i 2 \Pi \Omega_{\mathcal{F}_{C}}} e_{I P-j}\right)$ and

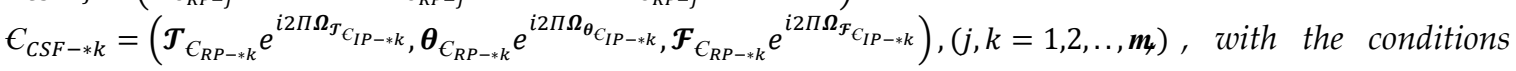

$\boldsymbol{T}_{R P-j} \geq \boldsymbol{T}_{R P-* k}, \boldsymbol{\Omega}_{\boldsymbol{T}_{I P-j}} \geq \boldsymbol{\Omega}_{\mathcal{T}_{I P-* k}}, \boldsymbol{\theta}_{R P-j} \leq \boldsymbol{\theta}_{R P-* k}, \boldsymbol{\Omega}_{\boldsymbol{\theta}_{I P-j}} \leq \boldsymbol{\Omega}_{\boldsymbol{\theta}_{I P-* k}}, \mathcal{F}_{R P-j} \leq \mathcal{F}_{R P-* k}$ and $\boldsymbol{\Omega}_{\mathcal{F}_{I P-j}} \leq \boldsymbol{\Omega}_{\mathcal{F}_{I P-* k}}$, we have $\operatorname{CSFBM} M^{S S F}, t_{C S F}\left(\epsilon_{C S F-1}, \epsilon_{C S F-2}, \ldots, \epsilon_{C S F-m_{4}}\right) \geq \operatorname{CSFBM} M^{S_{C S F}, t_{C S F}}\left(\epsilon_{C S F-* 1}, \epsilon_{C S F-* 2}, \ldots, \epsilon_{C S F-* m}\right)$.

Proof. Let $C S F B M^{S_{C S F}, t_{C S F}}\left(\epsilon_{C S F-1}, \epsilon_{C S F-2}, \ldots, \epsilon_{C S F-m}\right)=(u, v)$ and $\operatorname{CSFBM} M^{S_{C S F}, t_{C S F}}\left(\epsilon_{C S F-* 1}, \epsilon_{C S F-* 2}, \ldots, \epsilon_{C S F-* m}\right)=\left(u^{\prime}, v^{\prime}\right)$. The proof of the truth grade is to show that the real part is with $u^{\prime} \leq u$. If $\boldsymbol{T}_{\mathrm{RP}-\mathrm{j}} \geq \boldsymbol{T}_{\mathrm{RP}-* \mathrm{k}}, \boldsymbol{\Omega}_{\mathcal{T}_{\mathrm{IP}-\mathrm{j}}} \geq \boldsymbol{\Omega}_{\mathcal{T}_{\mathrm{IP}-* \mathrm{k}}}, \boldsymbol{\theta}_{\mathrm{RP}-\mathrm{j}} \leq \boldsymbol{\theta}_{\mathrm{RP}-* \mathrm{k}}, \boldsymbol{\Omega}_{\boldsymbol{\theta}_{\mathrm{IP}-\mathrm{j}}} \leq \boldsymbol{\Omega}_{\boldsymbol{\theta}_{\mathrm{IP}-* \mathrm{k}}}, \mathcal{F}_{\mathrm{RP}-\mathrm{j}} \leq \mathcal{F}_{\mathrm{RP}-* \mathrm{k}}$ and $\boldsymbol{\Omega}_{\mathcal{F}_{\mathrm{IP}-\mathrm{j}}} \leq \boldsymbol{\Omega}_{\mathcal{F}_{\mathrm{IP}-* \mathrm{k}}}$ then we have $\mathcal{T}_{* \mathrm{RP}-\mathrm{j}}^{\mathrm{S}_{\mathrm{CSF}}} \mathcal{T}_{* \mathrm{RP}-* \mathrm{k}}^{\mathrm{t}_{\mathrm{CSF}}} \mathrm{e}^{\mathrm{i} 2 \Pi\left(\boldsymbol{\Omega}_{* \mathcal{F}_{\mathrm{IP}-\mathrm{j}}}{ }^{\mathrm{S} C S F} \boldsymbol{\Omega}_{* \mathcal{F}_{\mathrm{IP}-* \mathrm{k}}}{ }^{\mathrm{t} C S F}\right)} \leq \mathcal{T}_{\mathrm{RP}-\mathrm{j}}^{\mathrm{S}} \mathcal{J}_{\mathrm{RP}-\mathrm{k}} \mathrm{e}^{\mathrm{i} 2 \Pi\left(\boldsymbol{\Omega}_{\mathcal{F}_{\mathrm{IP}-\mathrm{j}}}{ }^{\mathrm{s} C S F} \boldsymbol{\Omega}_{\mathcal{F}_{\mathrm{IP}-\mathrm{k}}}{ }^{\mathrm{t}}{ }_{\mathrm{CSF}}\right)}$,

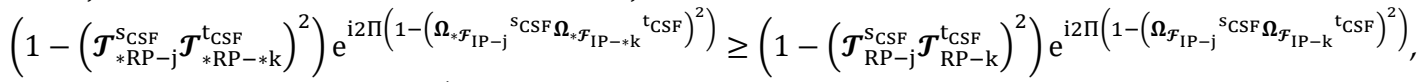




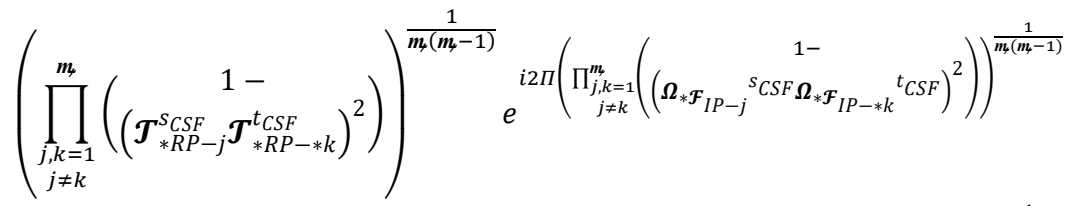

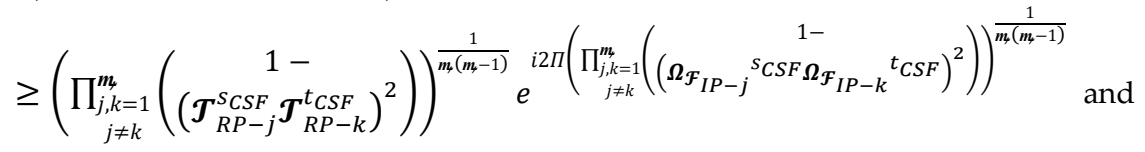

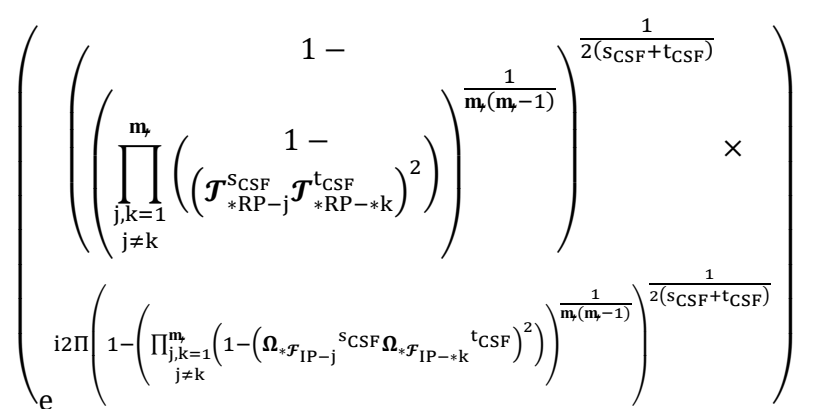

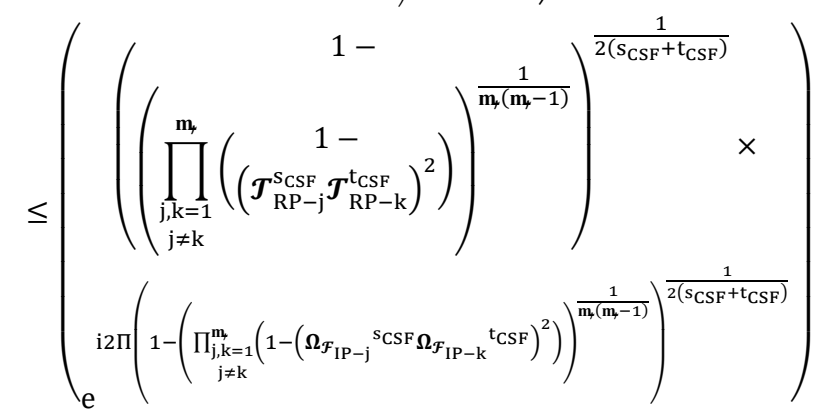

Hence, $u^{\prime} \leq u$. Similarly, $w^{\prime} \geq w, v^{\prime} \geq v$, for abstinence and falsity grades. Thus, we obtain the result with $\operatorname{CSFBM}^{S_{C S F}, t_{C S F}}\left(\epsilon_{C S F-1}, \epsilon_{C S F-2}, \ldots, \epsilon_{C S F-m_{r}}\right) \geq \operatorname{CSFBM}^{S_{C S F}, t_{C S F}}\left(\epsilon_{C S F-* 1}, \epsilon_{C S F-* 2}, \ldots, \epsilon_{C S F-* m}\right)$.

Theorem 4 (Boundedness). For any two CSFSs

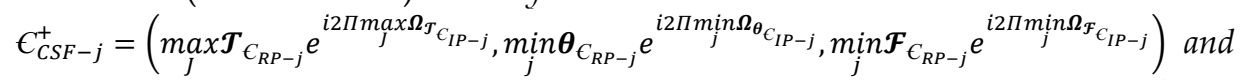

$\epsilon_{C S F-j}^{-}=\left(\min _{J} \boldsymbol{T}_{\epsilon_{R P-j}} e^{i 2 \Pi \min \boldsymbol{\Omega}_{\mathcal{T}_{I P-j}}}, \max _{j} \boldsymbol{\theta}_{\epsilon_{R P-j}} e^{i 2 \Pi \max _{j} \boldsymbol{\Omega}_{\boldsymbol{\theta}_{I P-j}}}, \max _{j} \mathcal{F}_{\epsilon_{R P-j}} e^{i 2 \Pi \max _{j} \boldsymbol{\Omega}_{\mathcal{F}_{\epsilon_{I P-j}}}}\right),\left(j=1,2, \ldots, \boldsymbol{m}_{\uparrow}\right)$, we obtain $\epsilon_{C S F-j}^{-} \leq C S F B M^{S_{C S F}, t_{C S F}}\left(\epsilon_{C S F-1}, \epsilon_{C S F-2}, \ldots, \epsilon_{C S F-m}\right) \leq \epsilon_{C S F-j}^{+}$.

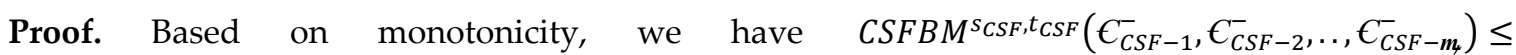

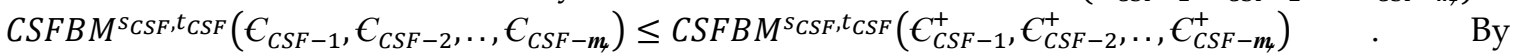

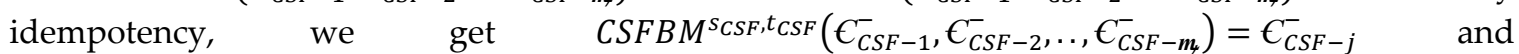
$\operatorname{CSFBM} M_{C S F}, t_{C S F}\left(C_{C S F-1}^{+}, \epsilon_{C S F-2}^{+}, \ldots, \epsilon_{C S F-m}^{+}\right)=\epsilon_{C S F-j}^{+}$. Then, we obtain the result with $\epsilon_{C S F-j}^{-} \leq$

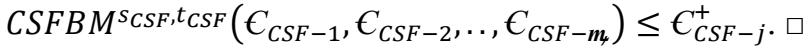

We previously have examined the three properties of idempotency, monotonicity, and boundedness for CSFSs. We next discuss more special cases with remarks.

Remark 1. When $t_{C S F}=0$ in Definition 6, we have $\operatorname{CSFBM} M^{S C S F, 0}\left(\epsilon_{C S F-1}, \epsilon_{C S F-2}, \ldots, \epsilon_{C S F-m}\right)=$ 


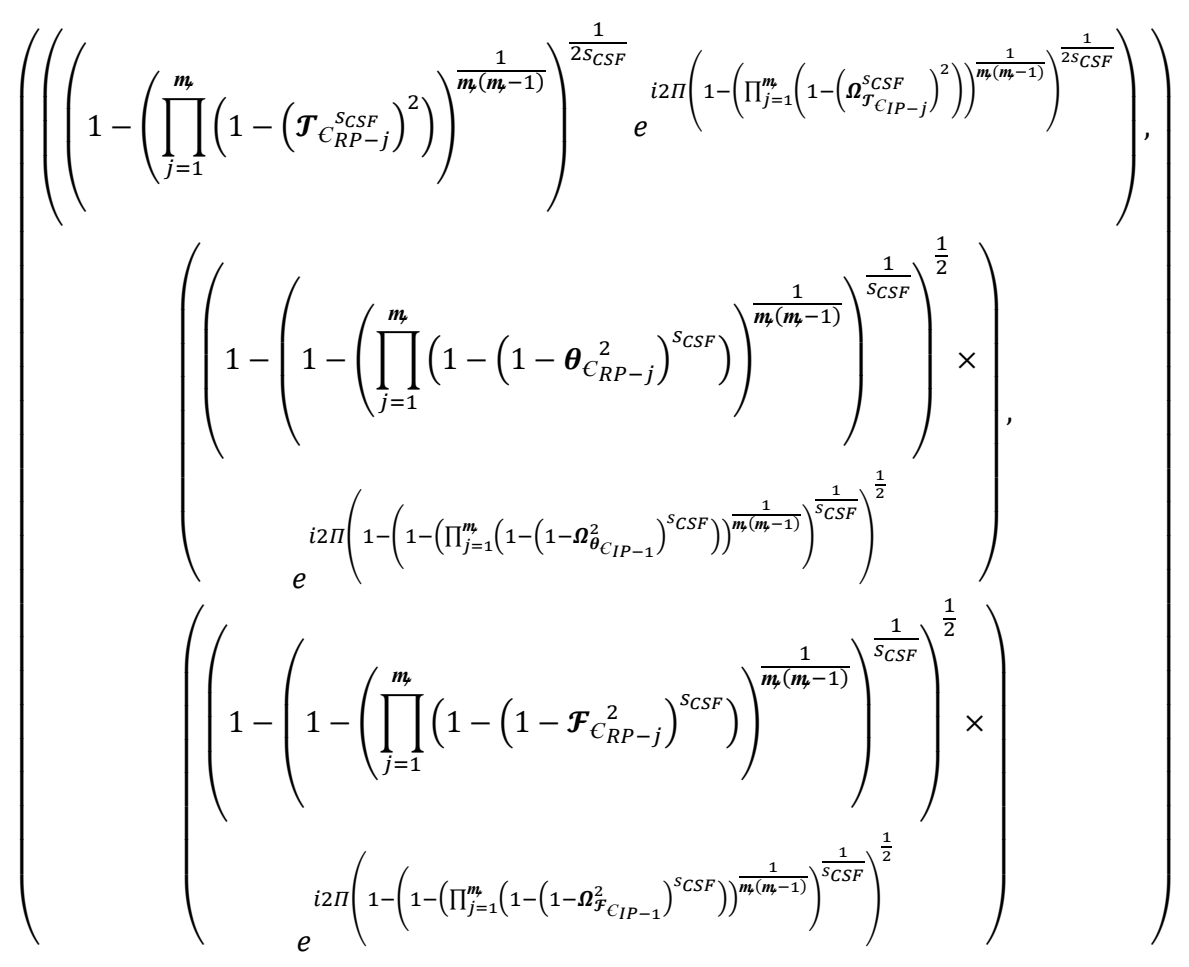

Remark 2. When $t_{C S F}=0, s_{C S F}=1$ in Definition 6, we have $\operatorname{CSFBM} M^{1,0}\left(\epsilon_{C S F-1}, \epsilon_{C S F-2}, \ldots, \epsilon_{C S F-m}\right)=$

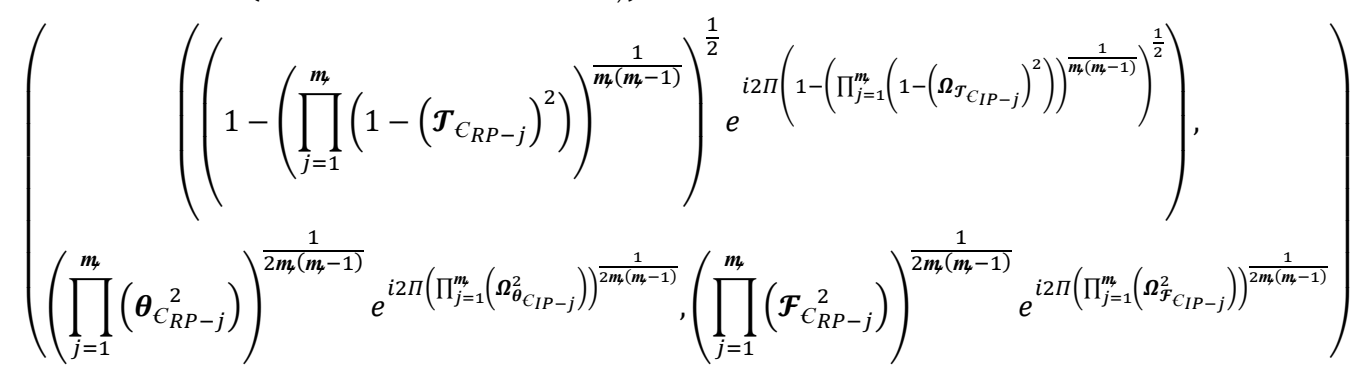

Remark 3. When $t_{C S F}=1, s_{C S F}=0$ in Definition 6, we have $\operatorname{CSFBM^{0,1}}\left(\epsilon_{C S F-1}, \epsilon_{C S F-2}, \ldots, \epsilon_{C S F-m}\right)=$

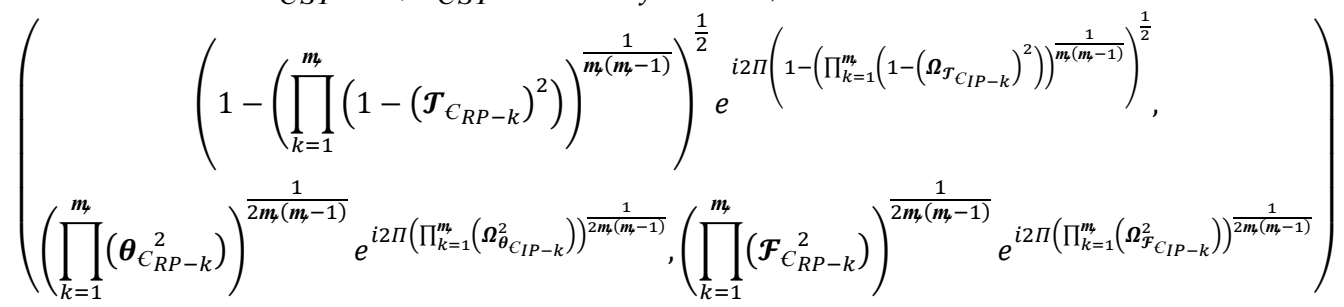

Remark 4. When $s_{C S F}=t_{C S F}=1$ in Definition 6, we have $\operatorname{CSFBM}^{1,1}\left(\epsilon_{C S F-1}, \epsilon_{C S F-2}, \ldots, \epsilon_{C S F-m}\right)=$ 
Mathematics 2020, 8, 1739

13 of 21

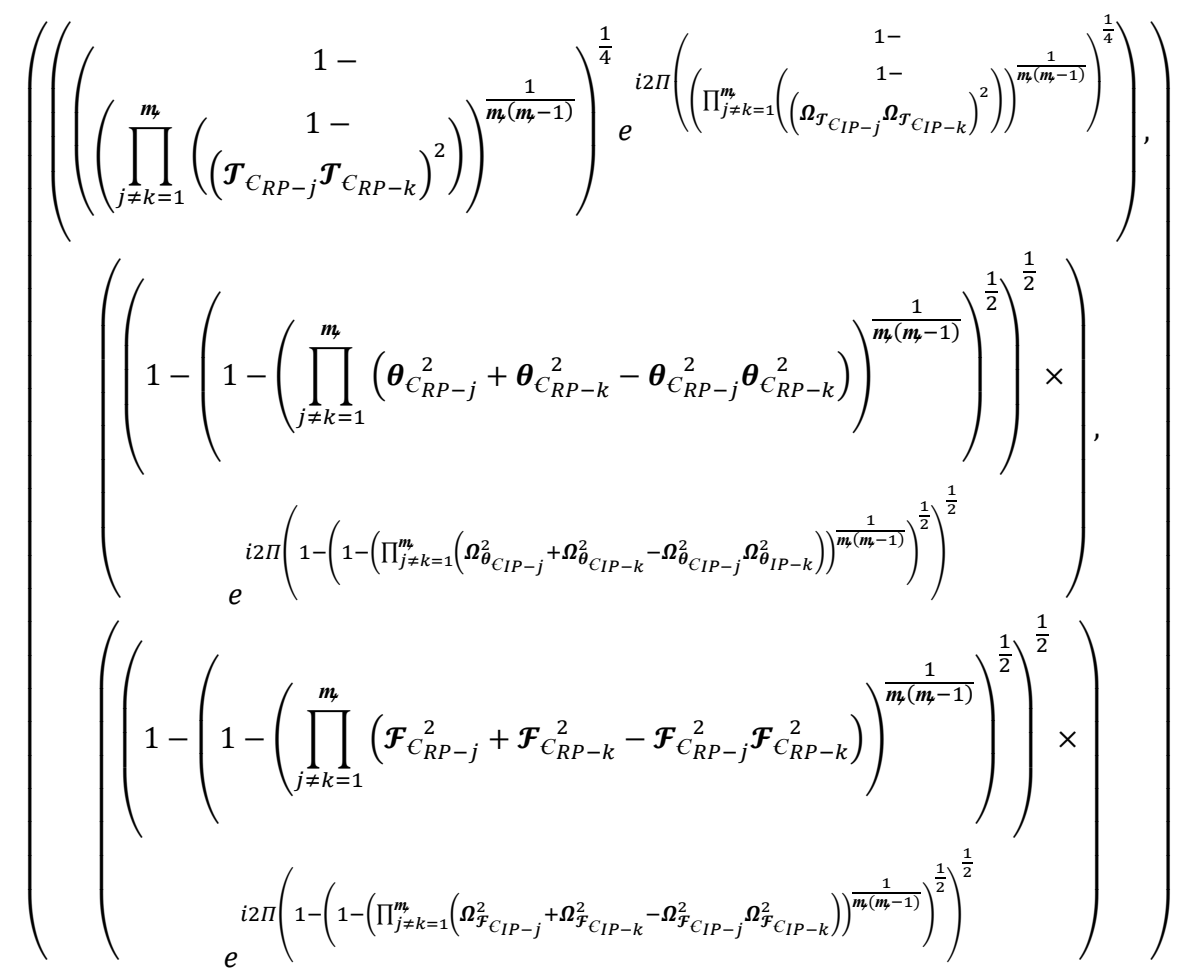

We next give the definition of the CSF-weighted-BM (CSFWBM) operator where the weight vector is expressed by $\mathrm{W}_{w}=\left(\mathrm{W}_{w-1}, \mathrm{~W}_{w-2}, \ldots, \mathrm{W}_{w-m_{4}}\right)^{T}$ with $\sum_{j=1}^{m_{w}} \mathrm{~W}_{w-j}=1$ and $\mathrm{W}_{\mathrm{w}-j} \in[0,1],(j=$ $\left.1,2, \ldots, \mathbf{m}_{4}\right)$.

Definition 7. For any CSFSS $\mathscr{C}_{C S F-j}, \mathbf{j}=\mathbf{1}, \mathbf{2}, \mathbf{3}, \ldots ., m$, the CSFWBM operator is defined as:

$$
\begin{aligned}
& \operatorname{CSFWBM}^{S_{C S F}, t_{C S F}}\left(\epsilon_{C S F-1}, \epsilon_{C S F-2}, \ldots, \epsilon_{C S F-m_{4}}\right) \\
& =\left(\frac{1}{m_{+}\left(m_{4}-1\right)} \bigoplus_{\substack{j, k=1 \\
j \neq k}}^{m_{k}}\left(\left(W_{w-j} \epsilon_{C S F-j}\right)^{s_{C S F}} \otimes\left(W_{w-k} \epsilon_{C S F-k}\right)^{t_{C S F}}\right)\right)^{\frac{1}{s_{C S F}+t_{C S F}}}
\end{aligned}
$$

Theorem 5. The aggregation result from Definition 7 is still a CSFS such that $C S F W B M^{s_{C S F}, t_{C S F}}\left(\mathrm{C}_{C S F-1}, \mathrm{C}_{C S F-2}, . ., \mathrm{C}_{C S F-m}\right)=$

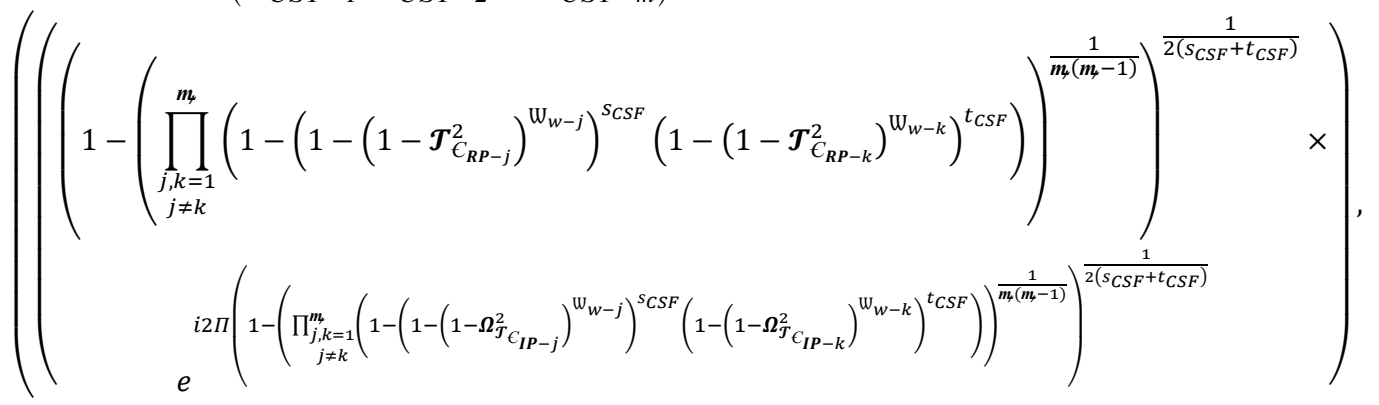




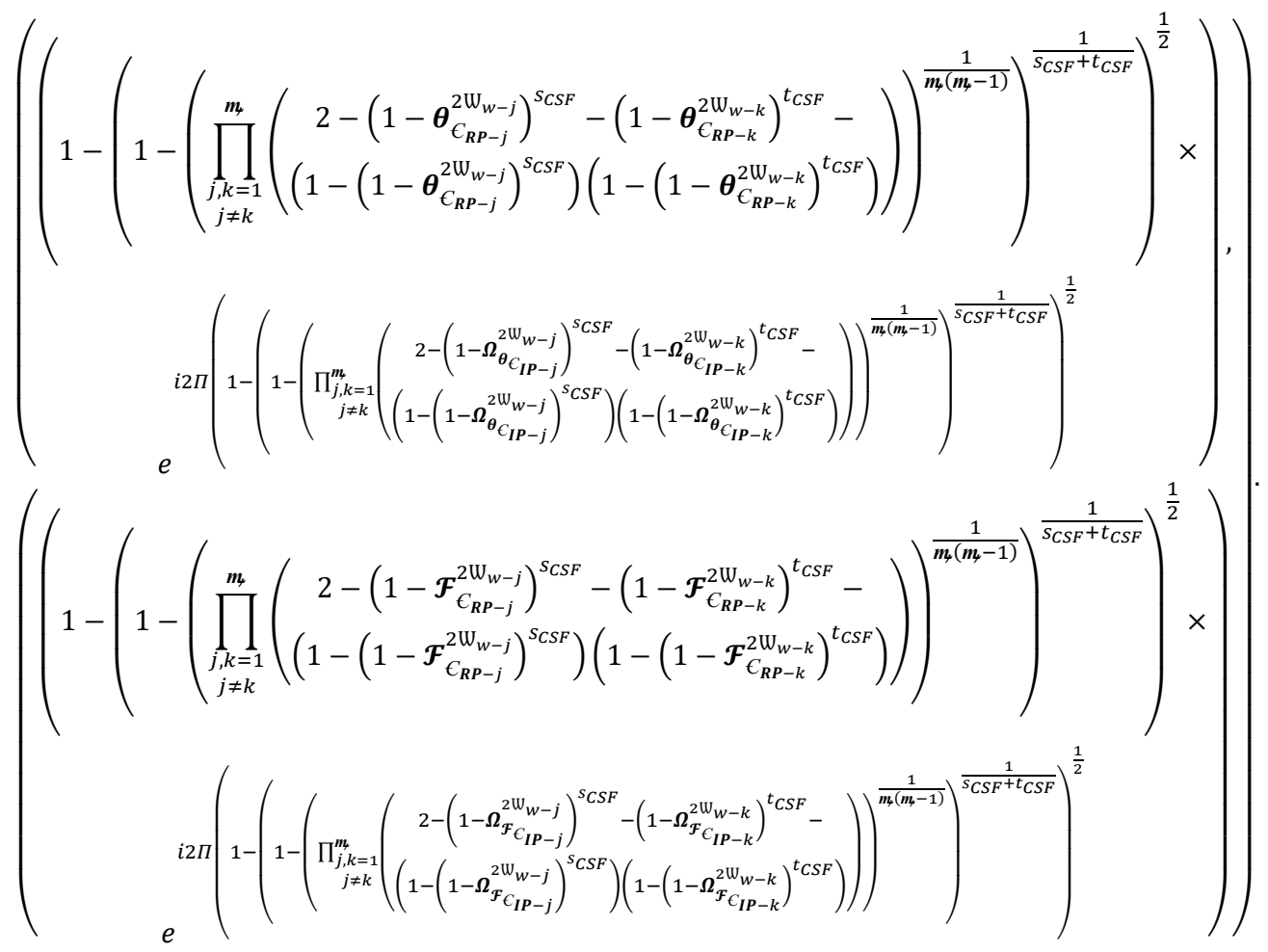

Proof. The proof of this theorem is similar to that of Theorem 1.

Similarly, we can obtain the properties of idempotency, monotonicity, and boundedness for the CSFWBM operator.

Theorem 6 (Idempotency). For any $C S F N \quad \mathrm{C}_{C S F-j}, j=1,2,3, \ldots ., \boldsymbol{m}_{\text {, }}$, we have $C S F W B M^{S C S F}, t_{C S F}\left(\mathrm{C}_{C S F-1}, \mathrm{C}_{C S F-2}, . ., \mathrm{C}_{C S F-} \boldsymbol{m}\right)=\mathrm{\epsilon}_{C S F}$.

Proof. The proof of this theorem is similar to that of Theorem 2 .

Theorem 7 (Monotonicity). For any two CSFNs

$\epsilon_{C S F-j}=\left(\boldsymbol{T}_{\epsilon_{R P-j}} e^{i 2 \Pi \Omega_{\mathcal{T}}} \epsilon_{I P-j}, \boldsymbol{\theta}_{\epsilon_{R P-j}} e^{i 2 \Pi \boldsymbol{\Omega}_{\boldsymbol{\theta}_{I P-j}}, \boldsymbol{F}_{\epsilon_{R P-j}} e^{i 2 \Pi \boldsymbol{\Omega}_{\mathcal{F}}} \epsilon_{I P-j}}\right)$ and $\epsilon_{C S F-* k}=$

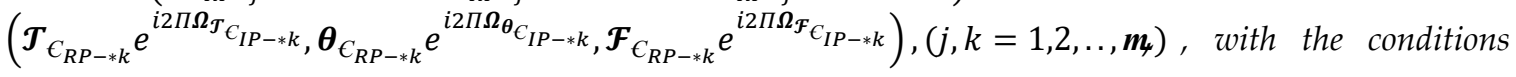
$\boldsymbol{T}_{R P-j} \geq \boldsymbol{T}_{R P-* k}, \boldsymbol{\Omega}_{\mathcal{T}_{I P-j}} \geq \boldsymbol{\Omega}_{\mathcal{T}_{I P-*} k^{\prime}}, \boldsymbol{\theta}_{R P-j} \leq \boldsymbol{\theta}_{R P-* k}, \boldsymbol{\Omega}_{\boldsymbol{\theta}_{I P-j}} \leq \boldsymbol{\Omega}_{\boldsymbol{\theta}_{I P-* k^{\prime}}} \boldsymbol{F}_{R P-j} \leq \mathcal{F}_{R P-* k} \quad$ and $\quad \boldsymbol{\Omega}_{\mathcal{F}_{I P-j}} \leq$ $\boldsymbol{\Omega}_{\mathcal{F}_{I P-* k}}$, we have $\operatorname{CSFWBM}{ }^{S C S F}, t_{C S F}\left(\epsilon_{C S F-1}, \epsilon_{C S F-2}, \ldots, \epsilon_{C S F-m}\right) \geq$ $\operatorname{CSFWBM} M^{S C S F}, t_{C S F}\left(\epsilon_{C S F-* 1}, \epsilon_{C S F-* 2}, \ldots, \epsilon_{C S F-* m_{4}}\right)$.

Proof. The proof of this theorem is similar to that of Theorem 3.

Theorem 8 (Boundedness). For any two CSFNs

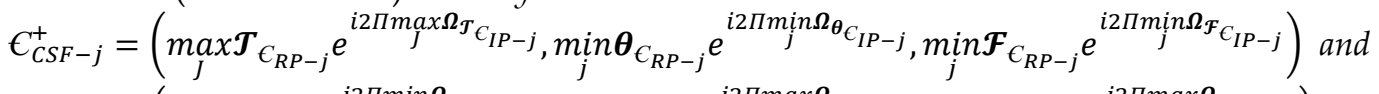

$$
\begin{aligned}
& \epsilon_{C S F-j}^{-}=\left(\min _{J} \boldsymbol{\mathcal { T }}_{\epsilon_{R P-j}} e^{i 2 \Pi \min _{j} \boldsymbol{\Omega}_{\mathcal{T}_{I P-j}}}, \max _{j} \boldsymbol{\theta}_{\epsilon_{R P-j}} e^{i 2 \Pi \max _{j} \boldsymbol{\Omega}_{\boldsymbol{\theta}_{I P-j}}, \max _{j} \mathcal{F}_{C_{R P-j}} e^{i 2 \Pi \max _{j} \boldsymbol{\Omega}_{\mathcal{F}}} \epsilon_{I P-j}}\right),(j= \\
& 1,2, \ldots, \boldsymbol{m}) \text {, we have that } \epsilon_{C S F-j}^{-} \leq C S F W B M^{S_{C S F}, t_{C S F}}\left(\epsilon_{C S F-1}, \epsilon_{C S F-2}, \ldots, \epsilon_{C S F-m}\right) \leq \epsilon_{C S F-j}^{+} \text {. }
\end{aligned}
$$

Proof. The proof of this theorem is similar to that of Theorem 4 . 


\section{MCDM and TOPSIS Methods Based on the CSFSs, CSFBM and CSFWBM operators}

In this section, we first use the proposed CSFBM and CSFWBM operators to solve MCDM problems. Furthermore, the Technique for Order Preference by Similarity to an Ideal Solution (TOPSIS) is an approach to identify an alternative that is closest to the positive ideal solution (PIS) and farthest from the negative ideal solution (NIS). We then consider the TOPSIS based on CSFSs and create a novel CSFS-TOPSIS.

\subsection{MADM Method Based on the Proposed Operators}

The aims of this subsection are to investigate MADM problems using the proposed CSFBM and CSFWBM operators. To resolve the MADM issues, we choose a family of alternatives and a family of attributes with respect to weight vectors to examine the reliability and proficiency of the proposed approaches, whose expressions are as follows: $€_{\mathrm{CSF}}=\left\{\mathrm{\epsilon}_{\mathrm{CSF}-1}, \mathrm{\epsilon}_{\mathrm{CSF}-2}, \mathrm{C}_{\mathrm{CSF}-3}, \ldots, \mathrm{\epsilon}_{\mathrm{CSF}-\mathrm{n}}\right\}$ and $\mathcal{L}_{A T}=$ $\left\{\mathcal{L}_{A T-1}, \mathcal{L}_{A T-2}, \ldots, \mathcal{L}_{A T-m}\right\}$ with the weight $\mathrm{W}_{w}=\left\{\mathrm{W}_{w-1}, \mathrm{~W}_{w-2}, \ldots, \mathrm{W}_{w-n}\right\}$ by using the complex spherical fuzzy information with $\epsilon_{\mathrm{CSF}}=\left(\boldsymbol{T}_{\epsilon_{R P-i j}} \mathrm{e}^{\mathrm{i} 2 \Pi \boldsymbol{\Omega}_{\mathrm{T}_{I P-i j}}, \boldsymbol{\theta}_{\epsilon_{R P-i j}}} \mathrm{e}^{\mathrm{i} 2 \Pi \boldsymbol{\Omega}_{\mathrm{G}_{I P-i j}}, \mathcal{F}_{\epsilon_{R P-i j}}} \mathrm{e}^{\mathrm{i} 2 \Pi \boldsymbol{\Omega}_{\mathcal{F}}} \epsilon_{I P-i j}\right)$. Thus, the steps of the proposed procedures are summarized as follows:

Step 1: Standardize the decision framework by using the following formula, if needed, then

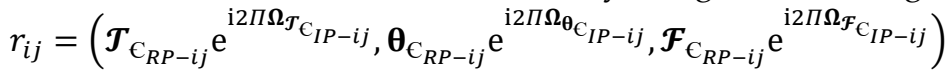

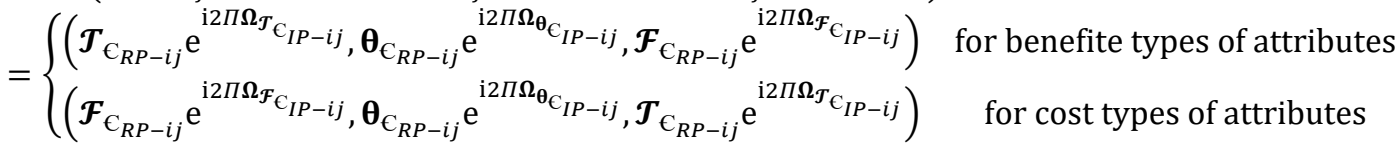

Step 2: By using the proposed operators of Equations (8) and (9), we investigate the complex spherical fuzzy number to aggregate the family of complex spherical fuzzy information.

Step 3: By using Equation (7), we investigate the score values of the aggregated values in Step 2.

Step 4: Rank all alternatives and find the best one.

To consider an application of the proposed MADM method, we next give an example which a venture organization needs to organize an expansion of income.

Example 1. If a venture organization needs to contribute to expand income, there are four possible organizations as choices, which are $\mathcal{L}_{A T-1}, \mathcal{L}_{A T-2}, \mathcal{L}_{A T-3}$, and $\mathcal{L}_{A T-4}$. There are five attributes used to assess options, including $\epsilon_{C S F-1}$ : the risk examination; $\epsilon_{C S F-2}$ : the development condition; $\epsilon_{C S F-3}$ : the social-political effect; $\epsilon_{C S F-4}$ : the environmental sway; $\epsilon_{C S F-5}$ : the advancement of the general public. To solve this problem, we choose the weight vector with $W_{w}=(0.3,0.2,0.15,0.35) T$. In fact, users can choose a weight vector according to their preference. Thus, the steps of the decision making procedures are summarized as follows:

Step 1: Standardize the decision framework by using the following formula

$$
\begin{aligned}
& r_{i j}=\left(\boldsymbol{T}_{\epsilon_{R P-i j}} \mathrm{e}^{\mathrm{i} 2 \Pi \boldsymbol{\Omega}_{\mathcal{T}}}{ }_{\epsilon_{I P-i j}}, \boldsymbol{\theta}_{\epsilon_{R P-i j}} \mathrm{e}^{\mathrm{i} 2 \Pi \boldsymbol{\Omega}_{\boldsymbol{\theta}}} \mathrm{C}_{I P-i j}, \mathcal{F}_{\mathrm{\epsilon}_{R P-i j}} \mathrm{e}^{\mathrm{i} 2 \Pi \boldsymbol{\Omega}_{\mathcal{F}}}{ }_{\epsilon_{I P-i j}}\right)
\end{aligned}
$$

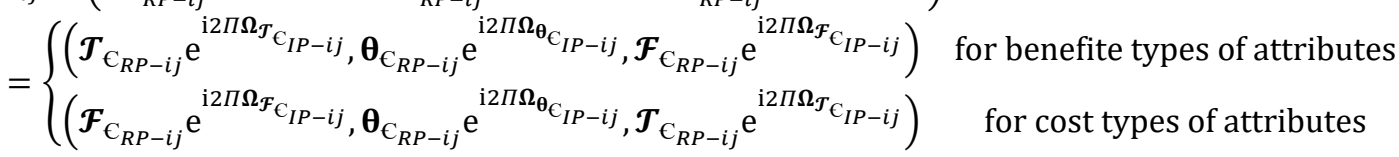

We obtain the decision matrix as shown in Table 1. We then go to the next Step 2. 
Table 1. Decision matrix.

\begin{tabular}{ccc}
\hline & $\mathcal{L}_{\boldsymbol{A} T-1}$ & $\mathcal{L}_{A T-2}$ \\
\hline $\mathscr{C}_{\text {CSF-1 }}$ & $\left(0.7 \mathrm{e}^{\mathrm{i} 2 \Pi(0.6)}, 0.3 \mathrm{e}^{\mathrm{i} 2 \Pi(0.2)}, 0.2 \mathrm{e}^{\mathrm{i} 2 \Pi(0.4)}\right)$ & $\left(0.71 \mathrm{e}^{\mathrm{i} 2 \Pi(0.61)}, 0.31 \mathrm{e}^{\mathrm{i} 2 \Pi(0.21)}, 0.21 \mathrm{e}^{\mathrm{i} 2 \Pi(0.41)}\right)$ \\
\hline $\mathscr{C}_{\text {CSF-2 }}$ & $\left(0.8 \mathrm{e}^{\mathrm{i} 2 \Pi(0.8)}, 0.2 \mathrm{e}^{\mathrm{i} 2 \Pi(0.2)}, 0.2 \mathrm{e}^{\mathrm{i} 2 \Pi(0.3)}\right)$ & $\left(0.81 \mathrm{e}^{\mathrm{i} 2 \Pi(0.81)}, 0.21 \mathrm{e}^{\mathrm{i} 2 \Pi(0.21)}, 0.21 \mathrm{e}^{\mathrm{i} 2 \Pi(0.31)}\right)$ \\
\hline $\mathscr{C}_{\text {CSF-3 }}$ & $\left(0.9 \mathrm{e}^{\mathrm{i} 2 \Pi(0.7)}, 0.1 \mathrm{e}^{\mathrm{i} 2 \Pi(0.2)}, 0.1 \mathrm{e}^{\mathrm{i} 2 \Pi(0.3)}\right)$ & $\left(0.91 \mathrm{e}^{\mathrm{i} 2 \Pi(0.71)}, 0.11 \mathrm{e}^{\mathrm{i} 2 \Pi(0.21)}, 0.11 \mathrm{e}^{\mathrm{i} 2 \Pi(0.31)}\right)$ \\
\hline $\mathscr{C}_{\text {CSF-4 }}$ & $\left(0.6 \mathrm{e}^{\mathrm{i} 2 \Pi(0.8)}, 0.5 \mathrm{e}^{\mathrm{i} 2 \Pi(0.3)}, 0.1 \mathrm{e}^{\mathrm{i} 2 \Pi(0.1)}\right)$ & $\left(0.61 \mathrm{e}^{\mathrm{i} 2 \Pi(0.81)}, 0.51 \mathrm{e}^{\mathrm{i} 2 \Pi(0.31)}, 0.11 \mathrm{e}^{\mathrm{i} 2 \Pi(0.11)}\right)$ \\
\hline $\mathscr{C}_{\text {CSF-5 }}$ & $\left(0.5 \mathrm{e}^{\mathrm{i} 2 \Pi(0.5)}, 0.4 \mathrm{e}^{\mathrm{i} 2 \Pi(0.4)}, 0.3 \mathrm{e}^{\mathrm{i} 2 \Pi(0.3)}\right)$ & $\left(0.51 \mathrm{e}^{\mathrm{i} 2 \Pi(0.51)}, 0.41 \mathrm{e}^{\mathrm{i} 2 \Pi(0.41)}, 0.31 \mathrm{e}^{\mathrm{i} 2 \Pi(0.31)}\right)$ \\
\hline $\mathscr{C}_{\text {CSF-1 }}\left(0.72 \mathrm{e}^{\mathrm{i} 2 \Pi(0.62)}, 0.32 \mathrm{e}^{\mathrm{i} 2 \Pi(0.22)}, 0.22 \mathrm{e}^{\mathrm{i} 2 \Pi(0.42)}\right)$ & $\left(0.73 \mathrm{e}^{\mathrm{i} 2 \Pi(0.63)}, 0.33 \mathrm{e}^{\mathrm{i} 2 \Pi(0.23)}, 0.23 \mathrm{e}^{\mathrm{i} 2 \Pi(0.43)}\right)$ \\
\hline $\mathscr{C}_{\text {CSF-2 }}$ & $\left(0.82 \mathrm{e}^{\mathrm{i} 2 \Pi(0.82)}, 0.22 \mathrm{e}^{\mathrm{i} 2 \Pi(0.22)}, 0.22 \mathrm{e}^{\mathrm{i} 2 \Pi(0.32)}\right)$ & $\left(0.83 \mathrm{e}^{\mathrm{i} 2 \Pi(0.83)}, 0.23 \mathrm{e}^{\mathrm{i} 2 \Pi(0.23)}, 0.23 \mathrm{e}^{\mathrm{i} 2 \Pi(0.33)}\right)$ \\
\hline $\mathscr{C}_{\text {CSF-3 }}$ & $\left(0.92 \mathrm{e}^{\mathrm{i} 2 \Pi(0.72)}, 0.12 \mathrm{e}^{\mathrm{i} 2 \Pi(0.22)}, 0.12 \mathrm{e}^{\mathrm{i} 2 \Pi(0.32)}\right)$ & $\left(0.93 \mathrm{e}^{\mathrm{i} 2 \Pi(0.73)}, 0.13 \mathrm{e}^{\mathrm{i} 2 \Pi(0.23)}, 0.13 \mathrm{e}^{\mathrm{i} 2 \Pi(0.33)}\right)$ \\
\hline $\mathscr{C}_{\text {CSF-4 }}$ & $\left(0.62 \mathrm{e}^{\mathrm{i} 2 \Pi(0.82)}, 0.52 \mathrm{e}^{\mathrm{i} 2 \Pi(0.32)}, 0.12 \mathrm{e}^{\mathrm{i} 2 \Pi(0.12)}\right)$ & $\left(0.63 \mathrm{e}^{\mathrm{i} 2 \Pi(0.83)}, 0.53 \mathrm{e}^{\mathrm{i} 2 \Pi(0.33)}, 0.13 \mathrm{e}^{\mathrm{i} 2 \Pi(0.13)}\right)$ \\
\hline $\mathscr{C}_{\text {CSF-5 }}$ & $\left(0.52 \mathrm{e}^{\mathrm{i} 2 \Pi(0.52)}, 0.42 \mathrm{e}^{\mathrm{i} 2 \Pi(0.42)}, 0.32 \mathrm{e}^{\mathrm{i} 2 \Pi(0.32)}\right)$ & $\left(0.53 \mathrm{e}^{\mathrm{i} 2 \Pi(0.53)}, 0.43 \mathrm{e}^{\mathrm{i} 2 \Pi(0.43)}, 0.33 \mathrm{e}^{\mathrm{i} 2 \Pi(0.33)}\right)$ \\
\hline
\end{tabular}

Step 2: By using the proposed operators of Equations (8) and (9), we investigate the complex spherical fuzzy number to aggregate the family of complex spherical fuzzy information, for $\mathrm{s}_{C S F}=$ $\mathrm{t}_{C S F}=1$. We obtain the aggregated values as shown in Table 2.

Table 2. Aggregated values by using Equations (8) and (9).

\begin{tabular}{ccc}
\hline Methods & CSFBM & CSFWBM \\
\hline $\mathscr{C}_{\text {CSF-1 }}$ & $\left(\begin{array}{c}0.3331 \mathrm{e}^{\mathrm{i} 2 \Pi(0.2111)}, \\
0.0925 \mathrm{e}^{\mathrm{i} 2 \Pi(0.041)}, \\
0.041 \mathrm{e}^{\mathrm{i} 2 \Pi(0.1626)}\end{array}\right)$ & $\left(\begin{array}{c}0.0005 \mathrm{e}^{\mathrm{i} 2 \Pi(0.0016)}, \\
0.0232 \mathrm{e}^{\mathrm{i} 2 \Pi(0.0098)}, \\
0.0098 \mathrm{e}^{\mathrm{i} 2 \Pi(0.043)}\end{array}\right)$ \\
\hline $\mathscr{C}_{\text {CSF-2 }}$ & $\left(\begin{array}{c}0.4957 \mathrm{e}^{\mathrm{i} 2 \Pi(0.4957)}, \\
0.041 \mathrm{e}^{\mathrm{i} 2 \Pi(0.041)}, \\
0.041 \mathrm{e}^{\mathrm{i} 2 \Pi(0.0925)}\end{array}\right)$ & $\left(\begin{array}{c}0.0001 \mathrm{e}^{\mathrm{i} 2 \Pi(0.0001)}, \\
0.0098 \mathrm{e}^{\mathrm{i} 2 \Pi(0.0098)}, \\
0.0098 \mathrm{e}^{\mathrm{i} 2 \Pi(0.0232)}\end{array}\right)$ \\
\hline $\mathscr{C}_{\text {CSF-3 }}$ & $\left(\begin{array}{c}0.7092 \mathrm{e}^{\mathrm{i} 2 \Pi(0.3331)}, \\
0.0102 \mathrm{e}^{\mathrm{i} 2 \Pi(0.041)}, \\
0.0102 \mathrm{e}^{\mathrm{i} 2 \Pi(0.0925)}\end{array}\right)$ & $\left(\begin{array}{c}0.0 \mathrm{e}^{\mathrm{i} 2 \Pi(0.0005)}, \\
0.0023 \mathrm{e}^{\mathrm{i} 2 \Pi(0.0098)}, \\
0.0023 \mathrm{e}^{\mathrm{i} 2 \Pi(0.00232)}\end{array}\right)$ \\
\hline $\mathscr{C}_{\text {CSF-4 }}$ & $\left(\begin{array}{c}0.2111 \mathrm{e}^{\mathrm{i} 2 \Pi(0.4957)}, \\
0.2493 \mathrm{e}^{\mathrm{i} 2 \Pi(0.0925)}, \\
0.0102 \mathrm{e}^{\mathrm{i} 2 \Pi(0.0102)}\end{array}\right)$ & $\left(\begin{array}{c}0.0016 \mathrm{e}^{\mathrm{i} 2 \Pi(0.0001)}, \\
0.0692 \mathrm{e}^{\mathrm{i} 2 \Pi(0.0232)}, \\
0.0023 \mathrm{e}^{\mathrm{i} 2 \Pi(0.0023)}\end{array}\right)$ \\
\hline $\mathscr{C}_{\text {CSF-5 }}$ & $\left(\begin{array}{c}0.1234 \mathrm{e}^{\mathrm{i} 2 \Pi(0.1234)}, \\
0.1626 \mathrm{e}^{\mathrm{i} 2 \Pi(0.1626)}, \\
0.0925 \mathrm{e}^{\mathrm{i} 2 \Pi(0.0925)}\end{array}\right)$ & $\left(\begin{array}{c}0.0037 \mathrm{e}^{\mathrm{i} 2 \Pi(0.0037)}, \\
0.043 \mathrm{e}^{\mathrm{i} 2 \Pi(0.043)}, \\
0.00232 \mathrm{e}^{\mathrm{i} 2 \Pi(0.0232)}\end{array}\right)$ \\
\hline
\end{tabular}


Step 3: By using Equation (6), we investigate the score values of the aggregated values in Step 2. The score values of the CSFBM operator are given as

$$
\begin{gathered}
S_{S F}\left(\epsilon_{C S F-1}\right)=0.0391, S_{S F}\left(\epsilon_{C S F-2}\right)=0.1593, S_{S F}\left(\epsilon_{C S F-3}\right)=0.2012, S_{S F}\left(C_{C S F-4}\right)=0.0731, S_{S F}\left(\epsilon_{C S F-5}\right) \\
=-0.013
\end{gathered}
$$

The score values of the CSFWBM operator are given as

$$
\begin{gathered}
S_{S F}\left(\epsilon_{C S F-1}\right)=-0.0009, S_{S F}\left(\epsilon_{C S F-2}\right)=-0.0003, S_{S F}\left(\epsilon_{C S F-3}\right)=-0.0002, S_{S F}\left(\epsilon_{C S F-4}\right)=-0.002, S_{S F}\left(\epsilon_{C S F-5}\right) \\
=-0.002
\end{gathered}
$$

Step 4: Rank all alternatives, and find the best one. The ranking results for the CSFBM operator are

$$
\epsilon_{C S F-3} \geq \epsilon_{C S F-2} \geq \epsilon_{C S F-4} \geq \epsilon_{C S F-1} \geq \epsilon_{C S F-5}
$$

The ranking results for the CSFWBM operator are

$$
\epsilon_{C S F-4} \geq \epsilon_{C S F-5} \geq \epsilon_{C S F-3} \geq \epsilon_{C S F-2} \geq \epsilon_{C S F-1}
$$

The two different operators give different results. The best alternative is $\epsilon_{C S F-3}$ using the CSFBM operator; the best alternative is $\epsilon_{C S F-4}$ using the CSFWBM operator.

\subsection{The TOPSIS Method Based on CSFSs}

We know that the TOPSIS is an approach to identify an alternative according to PIS and NIS. In this subsection, we construct the TOPSIS based on CSFSs, called a CSFS-TOPSIS. The steps of the CSF-TOPSIS method are as follows:

Step 1: First, we normalize the decision matrix based on CSFSs by considering the following formula:

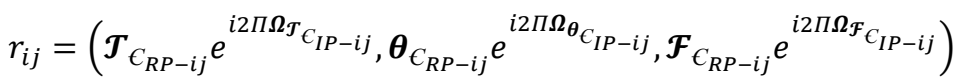

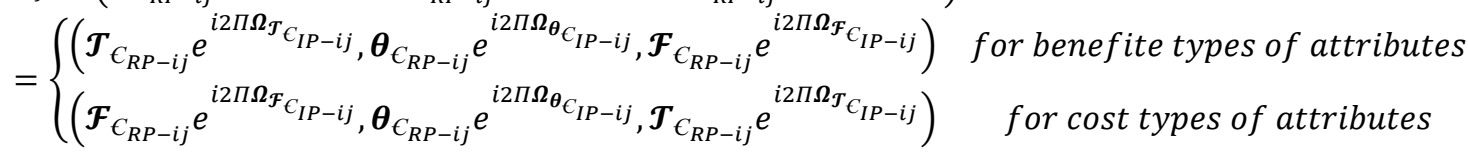

Step 2: By using the following Equations (10) and (11), we can examine the PIS and NIS among the alternatives.

$$
\begin{aligned}
& R^{+}=\left(r_{i 1}^{+}, r_{i 2}^{+}, r_{i 3}^{+}, \ldots, r_{i n}^{+}\right),
\end{aligned}
$$

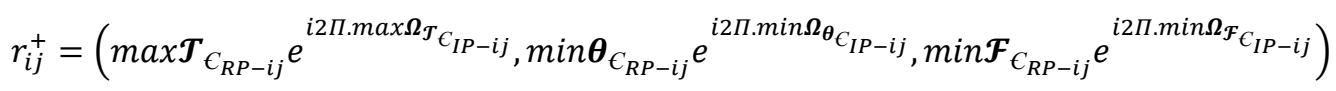

$$
\begin{aligned}
& R^{-}=\left(r_{i 1}^{-}, r_{i 2}^{-}, r_{i 3}^{-}, \ldots, r_{i n}^{-}\right), \\
& r_{i j}^{-}=\left(\min \boldsymbol{T}_{C_{R P-i j}} e^{i 2 \Pi \cdot \min \Omega_{\mathcal{T}}}{ }_{C_{I P-i j}, \max \boldsymbol{\theta}_{C_{R P-i j}}} e^{i 2 \Pi \cdot \max \boldsymbol{\Omega}_{\boldsymbol{\theta}_{C_{I P-i j}}}, \max \mathcal{F}_{C_{R P-i j}}} e^{i 2 \Pi \cdot \max \boldsymbol{\Omega}_{\mathcal{F}_{\mathcal{F}_{I P-i j}}}}\right)
\end{aligned}
$$

Step 3: Use the following Equation (12) to examine the complex spherical fuzzy PIS (CSF-PIS):

$$
\begin{aligned}
& K_{i}\left(r_{i j}, R^{+}\right)
\end{aligned}
$$

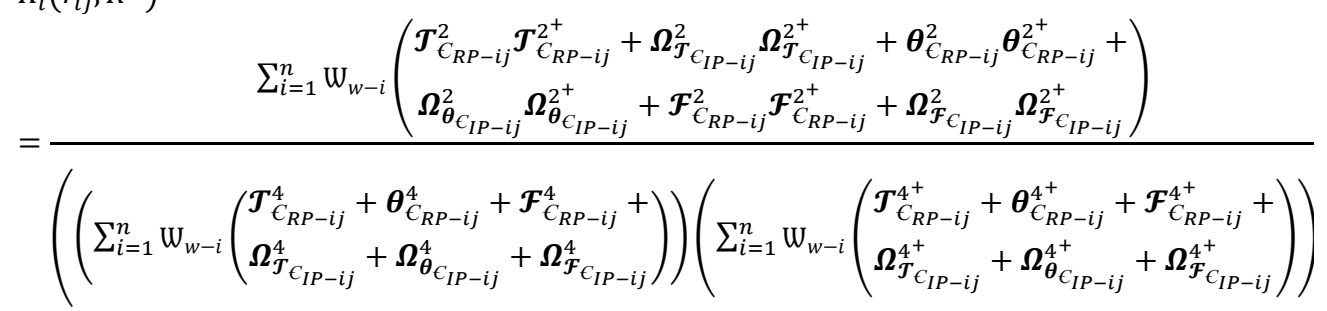


Examine the CSF-NIS by using the following Equation (13):

$$
\begin{aligned}
& K_{i}\left(r_{i j}, R^{-}\right)
\end{aligned}
$$

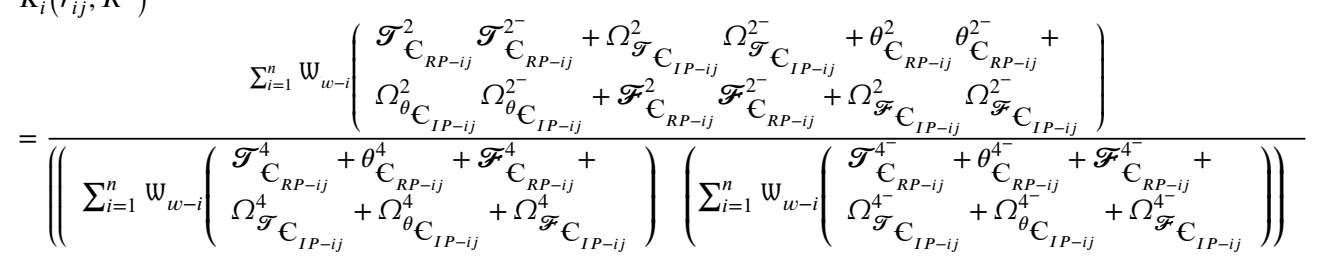

Step 5: Use the following Equation (14) to examine the closeness of each alternative:

$$
P_{i}=\frac{K_{i}\left(r_{i j}, R^{+}\right)}{K_{i}\left(r_{i j}, R^{+}\right)+K_{i}\left(r_{i j}, R^{-}\right)}
$$

Step 6: Rank all alternatives and choose the best one.

Based on the above proposed CSFS-TOPSIS method, we apply it to Example 1. Thus, we get the following results:

Step 1: According to the decision matrix based on CSFSs, we obtain the same decision matrix in Table 1, as Step 1 in the MADM method of Section 4.1, and so we go to step 2.

Step 2: By using Equations (10) and (11), we obtain the following PIS $R^{+}$and NIS $R^{-}$among the alternatives:

$$
\begin{aligned}
& R^{+} \\
& =\left(\begin{array}{c}
\left(0.9 \mathrm{e}^{\mathrm{i} 2 \Pi(0.8)}, 0.1 \mathrm{e}^{\mathrm{i} 2 \Pi(0.2)}, 0.1 \mathrm{e}^{\mathrm{i} 2 \Pi(0.1)}\right),\left(0.91 \mathrm{e}^{\mathrm{i} 2 \Pi(0.81)}, 0.11 \mathrm{e}^{\mathrm{i} 2 \Pi(0.21)}, 0.11 \mathrm{e}^{\mathrm{i} 2 \Pi(0.11)}\right), \\
\left(0.92 \mathrm{e}^{\mathrm{i} 2 \Pi(0.82)}, 0.12 \mathrm{e}^{\mathrm{i} 2 \Pi(0.22)}, 0.12 \mathrm{e}^{\mathrm{i} 2 \Pi(0.12)}\right),\left(0.93 \mathrm{e}^{\mathrm{i} 2 \Pi(0.83)}, 0.13 \mathrm{e}^{\mathrm{i} 2 \Pi(0.23)}, 0.13 \mathrm{e}^{\mathrm{i} 2 \Pi(0.13)}\right)
\end{array}\right) \\
& R^{-} \\
& =\left(\begin{array}{c}
\left(0.7 \mathrm{e}^{\mathrm{i} 2 \Pi(0.5)}, 0.5 \mathrm{e}^{\mathrm{i} 2 \Pi(0.4)}, 0.3 \mathrm{e}^{\mathrm{i} 2 \Pi(0.4)}\right),\left(0.71 \mathrm{e}^{\mathrm{i} 2 \Pi(0.51)}, 0.51 \mathrm{e}^{\mathrm{i} 2 \Pi(0.41)}, 0.31 \mathrm{e}^{\mathrm{i} 2 \Pi(0.41)}\right), \\
\left(0.72 \mathrm{e}^{\mathrm{i} 2 \Pi(0.52)}, 0.52 \mathrm{e}^{\mathrm{i} 2 \Pi(0.42)}, 0.32 \mathrm{e}^{\mathrm{i} 2 \Pi(0.42)}\right),\left(0.73 \mathrm{e}^{\mathrm{i} 2 \Pi(0.53)}, 0.53 \mathrm{e}^{\mathrm{i} 2 \Pi(0.43)}, 0.33 \mathrm{e}^{\mathrm{i} 2 \Pi(0.43)}\right)
\end{array}\right)
\end{aligned}
$$

Step 4: By using Equation (12), we obtain the following CSF-PIS:

$$
\begin{aligned}
K_{i}\left(\epsilon_{C S F-1}, R^{+}\right)= & 0.7887, K_{i}\left(\epsilon_{C S F-2}, R^{+}\right)=0.8849, K_{i}\left(\epsilon_{C S F-3}, R^{+}\right)=0.8967, K_{i}\left(\epsilon_{C S F-4}, R^{+}\right) \\
= & 0.7911, K_{i}\left(\epsilon_{C S F-5}, R^{+}\right)=0.4522
\end{aligned}
$$

Similarly, by using Equation (13), we obtain the following CSF-NIS:

$$
\begin{aligned}
K_{i}\left(\epsilon_{C S F-1}, R^{-}\right)= & 0.6647, K_{i}\left(\epsilon_{C S F-2}, R^{-}\right)=0.7346, K_{i}\left(\epsilon_{C S F-3}, R^{-}\right)=0.8178, K_{i}\left(\epsilon_{C S F-4}, R^{+}\right) \\
= & 0.5678, K_{i}\left(\epsilon_{C S F-5}, R^{+}\right)=0.3917
\end{aligned}
$$

Step 5: By using Equation (14), we obtain the following closeness of the alternatives:

$$
\mathrm{P}_{1}=0.5427, \mathrm{P}_{2}=0.5464, \mathrm{P}_{3}=0.5230, \mathrm{P}_{4}=0.5822, \mathrm{P}_{5}=0.5358
$$

Step 6: Rank all alternatives with

$$
P_{4} \geq P_{2} \geq P_{1} \geq P_{5} \geq P_{3}
$$

Thus, the best alternative is $\mathrm{P}_{4}$.

\subsection{Comparative Analysis}

Additionally, to investigate the reliability and effectiveness of the proposed operators, we chose some existing operators and compared with the proposed operators. The information on existing operators is as follows: Wang et al. [33] presented the aggregation operator based on PFS, and Ashraf and Abdullah [34] investigated the aggregation operator based on SFS and compared this with some special cases of the proposed approaches. The comparative analysis for the information in Table 1 on Example 1 is shown in Table 3. 
Table 3. Comparative analysis for the information in Table 1.

\begin{tabular}{|c|c|c|c|}
\hline Method & Operators & Score Values & Ranking \\
\hline \multirow{2}{*}{ Wang et al. [33] } & BM & Fail & Fail \\
\hline & WBM & Fail & Fail \\
\hline \multirow{2}{*}{$\begin{array}{c}\text { Ashraf and } \\
\text { Abdullah [34] }\end{array}$} & $\mathrm{BM}$ & Fail & Fail \\
\hline & WBM & Fail & Fail \\
\hline \multirow{2}{*}{ CPFS } & $\mathrm{BM}$ & Fail & Fail \\
\hline & WBM & Fail & Fail \\
\hline \multirow{2}{*}{$\begin{array}{l}\text { Proposed } \\
\text { method }\end{array}$} & $\mathrm{BM}$ & $\begin{aligned} & S_{S F}\left(€_{C S F-1}\right)= \\
& 0.0391, S_{S F}\left(€_{C S F-2}\right)=0.1593, \\
& S_{S F}\left(€_{C S F-3}\right)= \\
& 0.2012, S_{S F}\left(€_{C S F-4}\right)=0.0731 \\
& S_{S F}\left(€_{C S F-5}\right)=-0.013\end{aligned}$ & $\begin{array}{c}\mathrm{€}_{C S F-3} \geq \mathrm{C}_{C S F-2} \geq \mathrm{C}_{C S F-4} \\
\geq €_{C S F-1} \geq €_{C S F-5}\end{array}$ \\
\hline & WBM & $\begin{array}{c}S_{S F}\left(€_{C S F-1}\right)= \\
-0.0009, S_{S F}\left(€_{C S F-2}\right)=-0.0003 \\
S_{S F}\left(€_{C S F-3}\right)= \\
-0.0002, S_{S F}\left(€_{C S F-4}\right)=-0.002 \\
S_{S F}\left(\complement_{C S F-5}\right)=-0.002\end{array}$ & $\begin{array}{c}\mathrm{C}_{C S F-4} \geq \mathrm{C}_{C S F-5} \geq \mathrm{C}_{C S F-3} \\
\geq \mathrm{€}_{C S F-2} \geq €_{C S F-1}\end{array}$ \\
\hline
\end{tabular}

From the above analysis, we find that the existing operators of Wang et al. [33] with picture fuzzy BM and picture fuzzy WBM, and of Ashraf and Abdullah [34] with spherical fuzzy BM and spherical fuzzy WBM, fail to rank for the decision matrix in Table 1 . However, our proposed operators actually rank alternatives, with the best alternative $\mathrm{E}_{C S F-3}$ or $\mathrm{C}_{C S F-4}$, respectively.

\section{Conclusions}

In general, CSFSs are a mixture of CFSs and SFSs to cope with uncertain information in realistic decision-making issues, in which CSFSs consider the grades of truth, abstinence, and falsity, with a condition where the real part (and the imaginary part) of the three grades is not exceeded from a unit interval. In this paper, we investigate the operational laws of CSFSs with some properties. Additionally, based on CSFSs and Bonferroni mean (BM) operators, we construct two aggregation operators, called the complex spherical fuzzy Bonferroni mean (CSFBM) and the complex spherical fuzzy weighted Bonferroni mean (CSFWBM) operators. We also give the properties of idempotent, monotonicity, and boundedness for both CSFBM and CSFWBM operators. A MADM problem was chosen to be resolved based on the CSFBM and CSFWBM operators. We then propose the TOPSIS method based on CSFSs to construct the CSFS-TOPSIS method. To examine the effectiveness and reliability of the proposed methods, an application example is given to delineate the proposed approaches, with comparisons of existing methods. The results actually demonstrate that the proposed CSFBM and CSFWBM operators and CSFS-TOPSIS method are well suited to these fuzzy environments. In our future work, we will extend the proposed method to complex T-spherical fuzzy sets, complex neutrosophic sets, complex neutrosophic hesitant sets, and complex T-spherical hesitant fuzzy sets.

Author Contributions: Investigation, Z.A.; Methodology, T.M.; Supervision, M.-S.Y.; Writing of original draft, Z.A.; Writing of review \& editing, M.-S.Y. All authors have read and agreed to the published version of the manuscript.

Funding: This research received no external funding.

Conflicts of Interest: The authors declare no conflict of interest. 


\section{References}

1. Zadeh, L.A. Fuzzy sets. Inf. Control. 1965, 8, 338-353. [CrossRef]

2. Belohlavek, R.; Dauben, J.W.; Klir, G.J. Fuzzy Logic and Mathematics: A Historical Perspective; Oxford University Press: Oxford, UK, 2017.

3. Chakraverty, S.; Perera, S. Recent Advances in Applications of Computational and Fuzzy Mathematics; Springer: Berlin, Germany, 2018.

4. Pan, Y.; Zhang, L.; Li, Z.W.; Ding, L. Improved fuzzy Bayesian network-based risk analysis with interval-valued fuzzy sets and DS evidence theory. IEEE Trans. Fuzzy Syst. 2020, 28, 2063-2077. [CrossRef]

5. Atanassov, K. Intuitionistic fuzzy sets. Fuzzy Sets Syst. 1986, 20, 87-96. [CrossRef]

6. Yager, R.R.; Abbasov, A.M. Pythagorean membership grades, complex numbers, and decision making. Int. J. Intell. Syst. 2013, 28, 436-452. [CrossRef]

7. Garg, H. Linguistic Pythagorean fuzzy sets and its applications in multiattribute decision-making process. Int. J. Intell. Syst. 2018, 33, 1234-1263. [CrossRef]

8. Yang, M.S.; Hussain, Z. Fuzzy entropy for Pythagorean fuzzy sets with application to multicriterion decision making. Complexity 2018, 2018. [CrossRef]

9. Hussain, Z.; Yang, M.S. Distance and similarity measures of Pythagorean fuzzy sets based on Hausdorff metric with application to fuzzy TOPSIS. Int. J. Intell. Syst. 2019, 34, 2633-2654. [CrossRef]

10. Zhou, Q.; Mo, H.; Deng, Y. A new divergence measure of pythagorean fuzzy sets based on belief function and its application in medical diagnosis. Mathematics 2020, 8, 142. [CrossRef]

11. Yang, M.S.; Hussain, Z.; Ali, M. Belief and plausibility measures on intuitionistic fuzzy sets with construction of belief-plausibility TOPSIS. Complexity 2020, 2020. [CrossRef]

12. Faizi, S.; Salabun, W.; Rashid, T.; Zafar, S.; Watrobski, J. Intuitionistic fuzzy sets in multi-criteria group decision making problems using the characteristic objects method. Symmetry 2020, 12, 1382. [CrossRef]

13. Cuong, B.C.; Kreinovich, V. Picture fuzzy sets. J. Comput. Sci. Cybern. 2014, 30, 409.

14. Mahmood, T.; Ullah, K.; Khan, Q.; Jan, N. An approach toward decision-making and medical diagnosis problems using the concept of spherical fuzzy sets. Neural Comput. Appl. 2019, 31, 7041-7053. [CrossRef]

15. Ullah, K.; Hassan, N.; Mahmood, T.; Jan, N.; Hassan, M. Evaluation of investment policy based on multi-attribute decision-making using interval valued T-spherical fuzzy aggregation operators. Symmetry 2019, 11, 357. [CrossRef]

16. Ashraf, S.; Abdullah, S.; Abdullah, L. Child development influence environmental factors determined using spherical fuzzy distance measures. Mathematics 2019, 7, 661. [CrossRef]

17. Liu, P.; Khan, Q.; Mahmood, T.; Hassan, N. T-spherical fuzzy power Muirhead mean operator based on novel operational laws and their application in multi-attribute group decision making. IEEE Access 2019, 7, 22613-22632. [CrossRef]

18. Khan, M.J.; Kumam, P.; Deebani, W.; Kumam, W.; Shah, Z. Distance and similarity measures for spherical fuzzy sets and their applications in selecting mega projects. Mathematics 2020, 8, 519. [CrossRef]

19. Munir, M.; Kalsoom, H.; Ullah, K.; Mahmood, T.; Chu, Y.M. T-spherical fuzzy Einstein hybrid aggregation operators and their applications in multi-attribute decision making problems. Symmetry 2020, 12, 365. [CrossRef]

20. Ullah, K.; Garg, H.; Mahmood, T.; Jan, N.; Ali, Z. Correlation coefficients for T-spherical fuzzy sets and their applications in clustering and multi-attribute decision making. Soft Comput. 2020, 24, 1647-1659. [CrossRef]

21. Ramot, D.; Milo, R.; Friedman, M.; Kandel, A. Complex fuzzy sets. IEEE Trans. Fuzzy Syst. 2002, 10, $171-186$. [CrossRef]

22. Cacciola, M.; Calcagno, S.; Morabito, F.C.; Versaci, M. Swarm optimization for imaging of corrosion by impedance measurements in Eddy current test. IEEE Trans. Magn. 2007, 43, 1853-1856. [CrossRef]

23. Yazdanbakhsh, O.; Dick, S. A systematic review of complex fuzzy sets and logic. Fuzzy Sets Syst. 2018, 338, 1-22. [CrossRef]

24. Bi, L.; Zeng, Z.; Hu, B.; Dai, S. Two classes of entropy measures for complex fuzzy sets. Mathematics 2019, 7, 96. [CrossRef] 
25. Alkouri, A.M.D.J.S.; Salleh, A.R. Complex Intuitionistic Fuzzy Sets. In AIP Conference Proceedings; American Institute of Physics: College Park, MA, USA, 2012; Volume 1482, pp. 464-470.

26. Ullah, K.; Mahmood, T.; Ali, Z.; Jan, N. On some distance measures of complex Pythagorean fuzzy sets and their applications in pattern recognition. Complex Intell. Syst. 2020, 6, 15-27. [CrossRef]

27. Rani, D.; Garg, H. Complex intuitionistic fuzzy power aggregation operators and their applications in multicriteria decision-making. Expert Syst. 2018, 35, e12325. [CrossRef]

28. Yaqoob, N.; Gulistan, M.; Kadry, S.; Wahab, H.A. Complex intuitionistic fuzzy graphs with application in cellular network provider companies. Mathematics 2019, 7, 35. [CrossRef]

29. Garg, H.; Rani, D. Some results on information measures for complex intuitionistic fuzzy sets. Int. J. Intell. Syst. 2019, 34, 2319-2363. [CrossRef]

30. Ma, X.; Akram, M.; Zahid, K.; Alcantud, J.C.R. Group decision-making framework using complex Pythagorean fuzzy information. Neural Comput. Appl. 2020. [CrossRef]

31. Yager, R.R. On generalized Bonferroni mean operators for multi-criteria aggregation. Int. J. Approx. Reason. 2009, 50, 1279-1286. [CrossRef]

32. Ali, Z.; Mahmood, T.; Yang, M.S. Complex T-spherical fuzzy aggregation operators with application to multi-attribute decision making. Symmetry 2020, 12, 1311. [CrossRef]

33. Wang, C.; Zhou, X.; Tu, H.; Tao, S. Some geometric aggregation operators based on picture fuzzy sets and their application in multiple attribute decision making. Ital. J. Pure Appl. Math. 2017, 37, 477-492.

34. Ashraf, S.; Abdullah, S. Spherical aggregation operators and their application in multi-attribute group decision-making. Int. J. Intell. Syst. 2019, 34, 493-523. [CrossRef]

(C) 2020 by the authors. Licensee MDPI, Basel, Switzerland. This article is an open access article distributed under the terms and conditions of the Creative Commons Attribution (CC BY) license (http://creativecommons.org/licenses/by/4.0/). 